\title{
Granzyme B-induced cell death exerted by ex vivo CTL: discriminating requirements for cell death and some of its signs
}

\author{
J Pardo $^{1,2}$, R Wallich ${ }^{3}$, P Martin ${ }^{1}$, C Urban ${ }^{4}$, A Rongvaux ${ }^{5}$, RA Flavell ${ }^{6}$, A Müllbacher ${ }^{7}$, C Borner ${ }^{4}$ and MM Simon ${ }^{\star, 1}$
}

Granzyme B (gzmB) of cytotoxic T lymphocytes (CTL) is essential for recovery from intracellular pathogens, but the molecular basis of its action is still unresolved. Here, we analyzed gzmB-mediated death pathways under physiological conditions using ex vivo virus-immune CTLs that express perf and gzmB, but not gzmA (gzmB $\left.{ }^{+} \mathrm{CTL}\right)$. We show that $\mathrm{gzmB}^{+} \mathrm{CTL}$ abrogate target cell proliferation most likely by inducing cell death, independent of caspases and mitochondrial signaling. In addition, the data reveal that $\mathrm{gzmB}^{+} \mathrm{CTL}$ independently induce pro-apoptotic processes either via caspase-3/-7, leading to plasma membrane perturbance and ROS production or via Bid/Bak/Bax, resulting in cytochrome $c$ release and that both pathways elicit loss of $\Delta \Psi_{\mathrm{m}}$. Our data provide evidence for a pleiotropic pro-apoptotic function of gzmB presumably to counteract evasion strategies of pathogens and to control tumors.

Cell Death and Differentiation (2008) 15, 567-579; doi:10.1038/sj.cdd.4402289; published online 7 December 2007

Induction of programmed cell death (apoptosis) is a critical process in the host defense against intracellular pathogens and tumors. Natural killer (NK) cells and cytotoxic T lymphocytes (CTLs) are the main players in these processes. ${ }^{1}$ NK/CTL-induced apoptosis of infected or transformed target cells is mediated by the cytoplasmic granule components perforin (perf) and two abundant serine proteinases with highly distinct and restricted proteolytic activities, i.e. granzyme (gzm) A and gzmB. ${ }^{2,3}$ Previous studies have shown that gzmA and gzmB, when administered intracellularly together with perf, are able to induce, independently, various proapoptotic processes associated with target cell death. ${ }^{4,5}$ By employing purified gzmA/gzmB or CTL from mice deficient in gzmA and/or gzmB, it was demonstrated that mitochondrial damage is a key step in gzmA- and gzmB-induced apoptosis ${ }^{4-6}$ but that caspase activation only occurs in the presence of gzmB. ${ }^{4,6,7}$

Once released into the cytoplasm, gzmB was proposed to act via two mechanisms to trigger pro-apoptotic events: (1) to activate the effector caspase-3 by proteolytic processing ${ }^{8}$ and (2) to bypass caspase-3 activation and directly cleave cellular substrates implicated in the execution and/or morphology of apoptosis. $^{12}$ For the first mechanism, gzmB-activated caspase-3 cleaves dozens of cellular substrates, including the inhibitor ICAD which, upon degradation, releases the active endonuclease CAD for intranucleosomal degradation. In addition, active caspase-3 was shown to indirectly induce reactive oxygen species (ROS) production by targeting the p75 subunit of respiratory chain complexes I and II in previously permeabilized mitochondria. ${ }^{9}$ Through the action of cytosolic NADPH oxidase, active caspase-3 can also induce hydrogen peroxide and ROS production independent of mitochondria. ${ }^{10}$ Finally, caspase-3 activation by gzmB seems to be a key factor for the exposure of phosphatidylserine (PS) on the cell surface, one of several 'eat-me' signals for effective phagocytosis of apoptotic vesicles ${ }^{11}$ as well as for the cellular uptake of propidium iodide $(\mathrm{PI})$ due to increased plasma membrane permeability. ${ }^{6,8,12}$ Other caspases such as caspase-2, $-6,-7,-8,-9$ and -10 have been reported to serve as direct or indirect targets of gzmB, but may amplify/enhance rather than trigger CTL-mediated apoptosis. ${ }^{13}$

For the second mechanism, gzmB was found to directly cleave a number of structural and regulatory proteins of the cytoskeleton, cytosol and nucleus. ${ }^{4,5,8,14-17}$ This constitutes a caspase-independent gzmB trigger of CTL-mediated cell death, but the contribution of each substrate cleavage event to cell death remains poorly defined. A notable exception is the $\mathrm{BH} 3$-only protein Bid. This protein, belonging to the $\mathrm{Bcl}-2$ family of apoptosis regulators, can either be processed to truncated Bid (tBid) by caspase-8 in the death-receptor signaling pathway ${ }^{18}$ or, at an alternative cleavage site, by gzmB during CTL-mediated cell death. ${ }^{8,17}$ tBid is known to

\footnotetext{
${ }^{1}$ Metschnikoff Laboratory, Max-Planck-Institut für Immunbiologie, Freiburg, Germany; ${ }^{2}$ Dpto. Bioquímica y Biología Molecular y Celular, Facultad de Ciencias, Universidad de Zaragoza, Zaragoza, Spain; ${ }^{3}$ Institut für Immunologie, Universitätsklinikum Heidelberg, Heidelberg, Germany; ${ }^{4}$ Institute of Molecular Medicine and Cell Research, Center for Biochemistry and Molecular Research, Freiburg, Germany; ${ }^{5}$ Department of Immunobiology, Yale University School of Medicine, New Haven, CT, USA; ${ }^{6}$ Section of Immunobiology, Howard Hughes Medical Institute, Yale University School of Medicine, New Haven, CT, USA and ${ }^{7}$ Viral Immunology, Division of Immunology and Genetics, John Curtin School of Medical Research, Australian National University, Canberra, Australia

${ }^{*}$ Corresponding author: MM Simon, Metschnikoff Laboratory, Max-Planck-Institut für Immunbiologie, Stübeweg 51, Freiburg, Germany.

Tel: +49 7615108 533; Fax: + 497615108 529; E-mail: simon@immunbio.mpg.de

Keywords: CTL; granzyme B; caspases; mitochondria; apoptosis

Abbreviations: B6, C57BL/6; Casp, caspase; CTL, cytotoxic T lymphocyte; $\mathrm{DiOC}_{6}(3), 3,3^{\prime}$-dihexyloxacarbocyanine iodide; gzm, granzyme; 2-HE, dihydroethidium; LCMV, lymphocytic choriomeningitis virus; MEF, mouse embryonic fibroblast; $\Delta \Psi_{\mathrm{m}}$, mitochondrial transmembrane potential; NK, natural killer; perf, perforin; PI, propidium iodide; PS, phosphatidylserine; ROS, reactive oxygen species

Received 08.5.07; revised 20.9.07; accepted 29.10.07; Edited by SJ Martin; published online 07.12.07
} 
translocate to mitochondria where it activates Bax and/or Bak, two pro-apoptotic members of the Bcl-2 family that mediate increased mitochondrial membrane permeability and the release of apoptogenic factors, such as cytochrome $c$, apoptosis-inducing factor (AIF) and endonuclease $\mathrm{G}$ (endoG) from the intermembrane space into the cytosol. ${ }^{19}$ While the latter two factors trigger caspase-independent death signaling pathways, cytochrome $c$ binds to Apaf-1 and recruits caspase-9 into a high molecular weight heptameric structure called the apoptosome, which is then responsible for activating effector caspase- 3 and -7 . In contrast to the direct processing and activation of caspase-3, this $\mathrm{tBid} / \mathrm{Bax} / \mathrm{Bak}$ mediated mitochondrial pathway would be an indirect mode of action of gzmB to activate caspase-3 and -7.

At present, there is some controversy regarding the interplay of these two modes of actions of gzmB as well as the exact order of the events triggered by this protease during CTL-mediated apoptosis. The main focus of research has been directed toward mechanisms of how gzmB initiates mitochondrial damage. One pathway is thought to involve the direct action of gzmB on mitochondria to provoke suppression of mitochondrial transmembrane potential $\left(\Delta \Psi_{\mathrm{m}}\right)$ and increased ROS production without the release of apoptogenic factors such as cytochrome $c .{ }^{20}$ Another pathway was shown to require gzmB-mediated Bid cleavage to release cytochrome $c$ as well as to trigger $\Delta \Psi_{\mathrm{m}}$ loss and/or ROS production. ${ }^{17}$ Moreover, for the concomitant induction of AIF and endoG release from mitochondria by $\mathrm{gzmB}$ and $\mathrm{a}$ sustained $\Delta \Psi_{\mathrm{m}}$ loss, additional activation of caspases seems to be required. ${ }^{12}$ Finally, others have suggested that neither Bid nor Bak and Bax are necessary for initiating mitochondrial perturbation, ${ }^{7,21}$ but that caspases $^{21}$ or other cytosolic factors $^{7}$ are needed. Recent evidence from caspase-3/-7 double knockout cells has lent support to the notion that effector caspases can be critical mediators of mitochondrial events during apoptosis, ${ }^{22}$ but it has remained enigmatic to what extent they play a role in gzmB-initiated mitochondrial signaling leading to cell death. ${ }^{17,21}$

The major hurdle for dissecting the molecular actions of gzmB during CTL-mediated cell death has been the use of artificial in vitro or semi-intact cellular systems with purified gzmB, gzmA and perf preparations rather than cell-based CTL assays. Here, we have used physiological conditions namely ex vivo virus-immune CTLs that express perf and gzmB, but not gzmA (gzmB ${ }^{+} \mathrm{CTL}$ ) or any other functionally active gzm to reevaluate the pro-apoptotic potential of gzmB under more physiological conditions.

\section{Results}

Experimental system. CTLs were ex vivo-derived virusimmune $\mathrm{CD}^{+} \mathrm{T}$ cells from $\mathrm{gzmA}^{-1-}$ mice 8 days after lymphocytic choriomeningitis virus (LCMV) infection. These cells express perf and gzmB but not gzmA $\left(g^{2} m B{ }^{+} \mathrm{CTL}\right.$; Supplementary Figure S1). As target cells we used mouse embryonic fibroblasts (MEFs) derived from wild-type (MEF.wt) and knockout (ko) mutant mice with deficiencies of either, Bid (MEF.Bid ${ }^{-1-}$ ), Bak $\times$ Bax (MEF.Bak $\left.\times \mathrm{Bax}^{-1-}\right)$, caspase-3 (MEF.Casp $3^{-1-}$ ), caspase-7 (MEF.Casp $7^{-1-}$ ) or both (MEF.Casp $3 \times 7^{-/-}$) or of caspase-9 (MEF.Casp $9^{-1-}$ ).

As previously reported, ${ }^{6} \mathrm{gzmB}^{+} \mathrm{CTL}$ contained similar percentages of $g p 33 / D^{b}$ pentamer ${ }^{+} T$ cells to those from B6 (C57BL/6), gzmA $\times \mathrm{B}^{-/-}$and perf ${ }^{-1-}$ mice (8 versus 7,10 and $11 \%$, respectively; Supplementary Figure $S 1 a)$. The levels of gzmB were also roughly the same in gzmB ${ }^{+}$CTL, B6 CTL and perf $^{-1-}$ CTL and not detectable in gzmA $\times \mathrm{B}^{-1-} \mathrm{CTL}$ (Supplementary Figure $S 1 b)$. All four ex vivo CTL populations, independent of their genotype, expressed gzmK transcripts, but lacked mRNA for gzmC, D, E, F, G and M, with the exception of perf ${ }^{-1-}$ CTL, which expressed low levels of gzmC transcripts (Supplementary Figure S1d).

gzmB ${ }^{+}$CTLs have a similar potential as B6 CTL to induce cell death (Pardo et al. ${ }^{6}$ and data not shown), loss of plasma membrane integrity (monitored by exposure of PS at the plasma membrane and incorporation of $\mathrm{PI}$ ) and severe mitochondrial perturbation (monitored by reduction of the $\Delta \Psi_{\mathrm{m}}$ and increase in the generation of ROS) when tested on gp33-pulsed MEF.wt cells (Supplementary Figure S2a, b). These data indicate that CTL/target cell interaction and subsequent exocytosis is normal in $\mathrm{gzmB}^{+} \mathrm{CTL}$. They also confirm previous results with EL4 target cells ${ }^{6}$ showing that CTL-induced loss of plasma membrane and mitochondrial integrity as well as cell death is readily elicited by gzmB in the absence of gzmA. In contrast to $g z m B^{+} \mathrm{CTL}$, both gzmA $\times \mathrm{B}^{-/-}$and perf ${ }^{-/-} \mathrm{CTL}$ showed only marginal, if any, pro-apoptotic manifestations (Supplementary Figure S3a, b), and were unable to kill MEF.wt cells, as determined by a cell survival assay (Supplementary Figure S3c; and Pardo et al. ${ }^{6}$ ). However, all three CTL populations were similarly able to mobilize Lamp-1 to the cell surface after incubation with gp33labeled MEF.wt cells (Supplementary Figure S3d). These data exclude any contribution from additional proteases, including gzmK (transcripts expressed in all wt and gzmA/B ko strains; see above), to the pro-apoptotic activities of $\mathrm{gzmB}^{+} \mathrm{CTL}$ and suggest that the intracellular processes induced by $\mathrm{gzmB}^{+} \mathrm{CTL}$ in MEF.wt cells, including cell death, are exclusively due to gzmB.

As different MEF targets were used, we determined whether all mutant MEFs had the same ability as MEF.wt cells to induce degranulation of $\mathrm{gzmB}^{+} \mathrm{CTL}$ upon antigen recognition. For this purpose, degranulation of $\mathrm{gzmB}^{+} \mathrm{CTL}$ was determined by monitoring expression of the lysosomal marker Lamp-1 on their plasma membrane, following incubation with MEF.wt cells in the presence or absence of gp33. As shown in Supplementary Figure S1c, similar expression levels of Lamp-1 were seen in gzmB ${ }^{+} \mathrm{CTL}$ after incubation with any of the gp33-pulsed, but not mock-treated MEF lines.

Bid and Bak/Bax are critical for gzmB $^{+}$CTL-induced cytochrome $c$ release, but not for plasma membrane perturbation, mitochondrial depolarization or target cell killing. We first tested which of the pro-apoptotic effects induced by $\mathrm{gzmB}^{+} \mathrm{CTL}$ are governed through the Bid/Bak/ Bax-mediated mitochondrial axis in our experimental system. As shown in Figure 1, PS exposure and PI incorporation (Figure $1 \mathrm{a}$ and $\mathrm{c}$ ), as well as reduction of $\Delta \Psi_{\mathrm{m}}$ and generation of ROS (Figure $1 b$ and $d$ ), were elicited to the same extent by $\mathrm{gzmB}^{+} \mathrm{CTL}$ in MEF.wt and both koMEF 

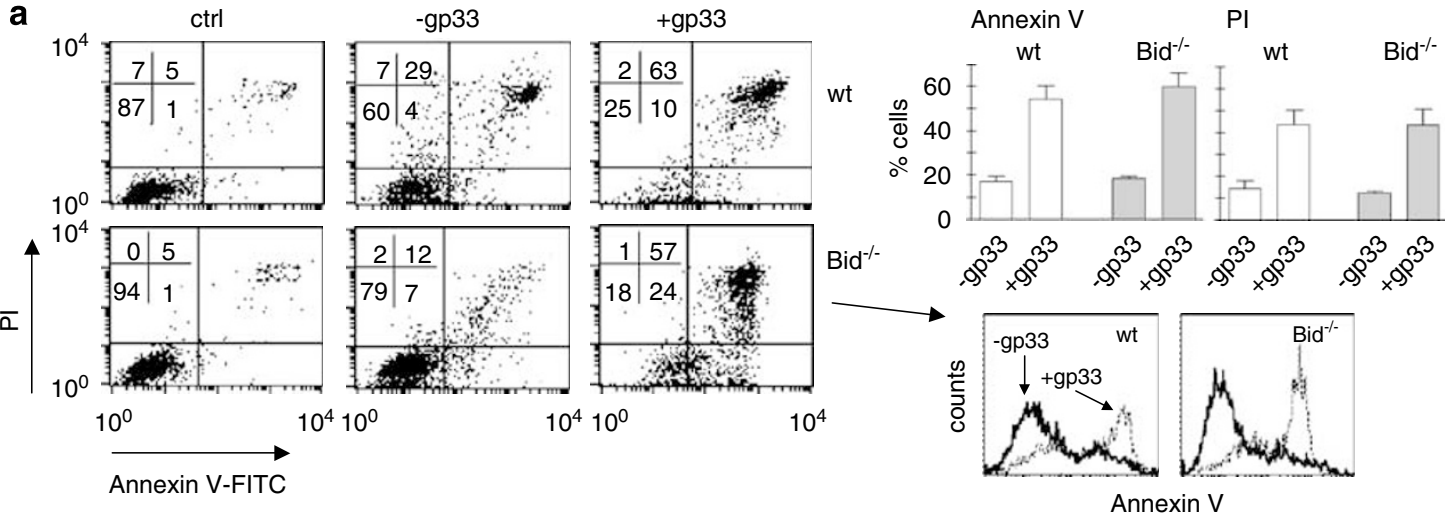

b
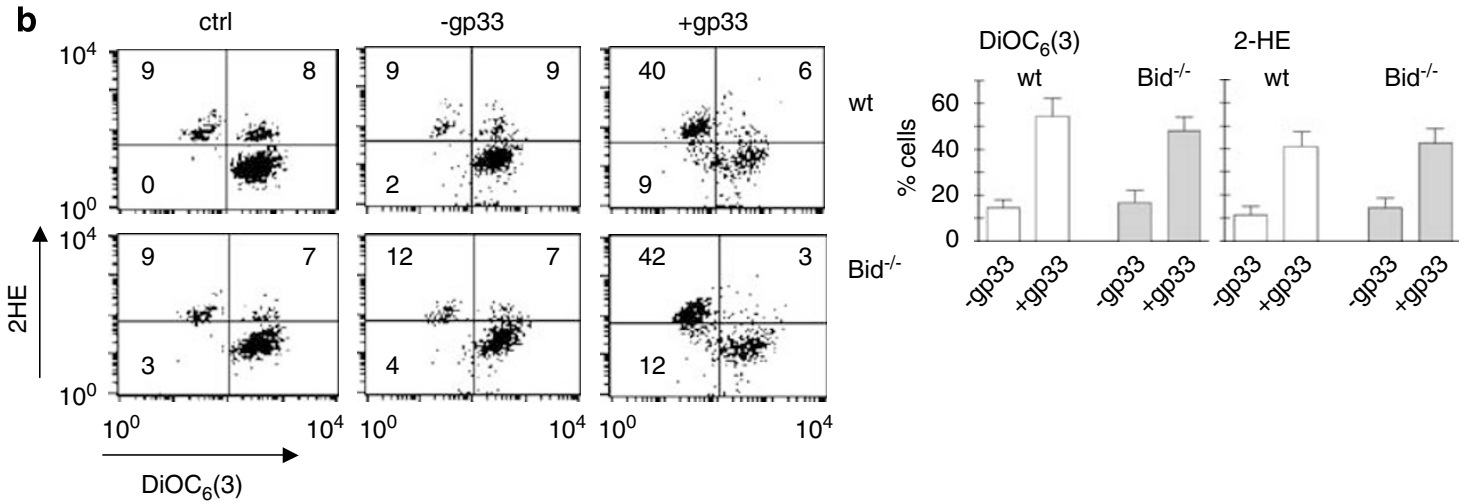

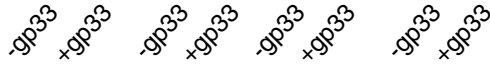

C

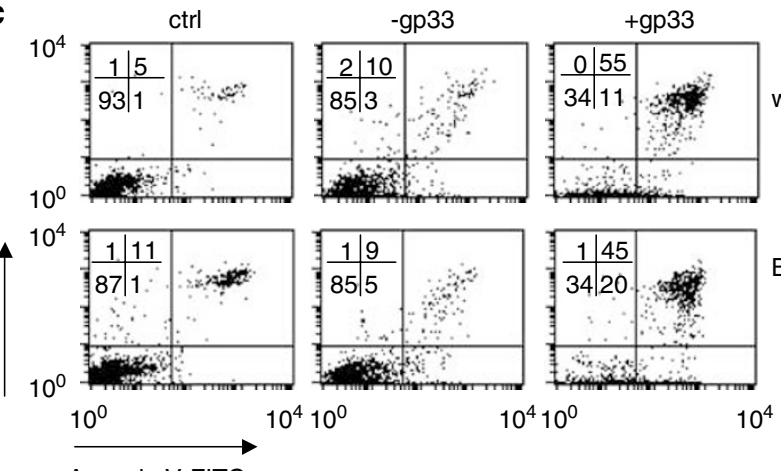

Annexin V-FITC
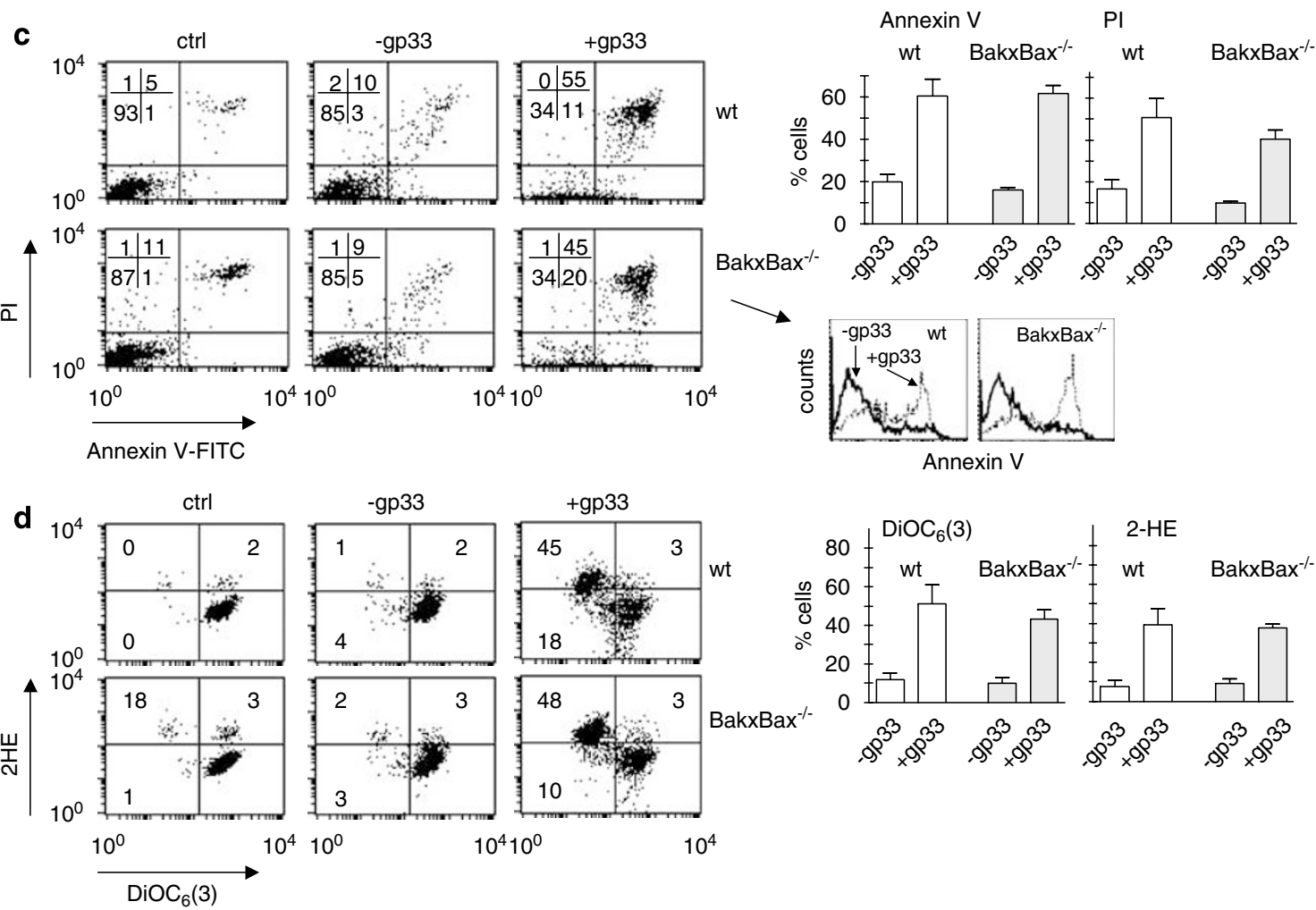

Figure $1 \mathrm{gzmB}^{+}$CTLs induce membrane and mitochondrial perturbation in the absence of either Bid or Bak and Bax. Gp33-pulsed MEF.wt, MEF.Bid ${ }^{-/-}(\mathbf{a}, \mathbf{b})$ and MEF.Bak $\times \operatorname{Bax}^{-1-}$ (c, d) cells were incubated with ex vivo virus-immune gzmB ${ }^{+}$CTL (MACS selected, $95 \%$ CD8 $^{+}$cells) $(1 \mathrm{~h}$ and/or $4 \mathrm{~h}, 10: 1$ effector/target ratio).

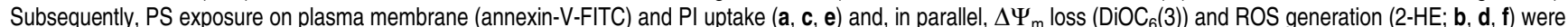
analyzed by three-color flow cytometry in the cell population negative for CD8 expression as described in Materials and Methods. A representative experiment is shown in the left panels. Numbers correspond to the percentage of cells in each quadrant. Data in the right panels are represented as the mean \pm S.E.M. of six (a, b), five (c) and four (d) independent experiments, respectively. (a, c) Histograms corresponding to the annexin-V staining in the dot plots are shown 
e

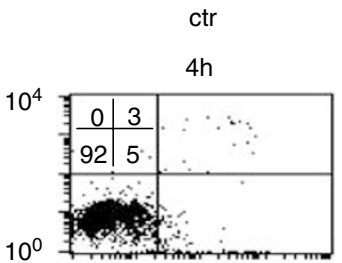

$10^{4}$

$10^{\circ}$
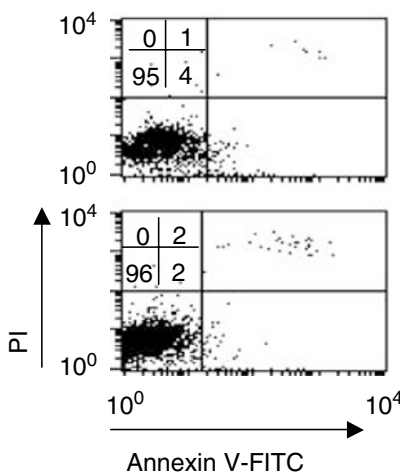

$\mathbf{f}$

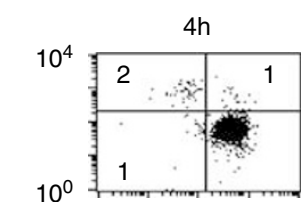

$10^{4}$
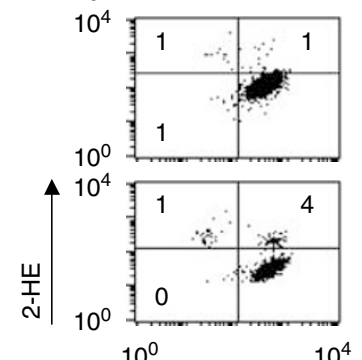

$\stackrel{10^{0}}{\stackrel{10^{4}}{\longrightarrow}}$ -gp33
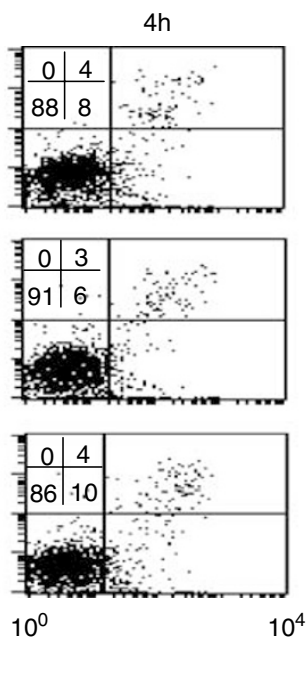

-gp33

$4 \mathrm{~h}$
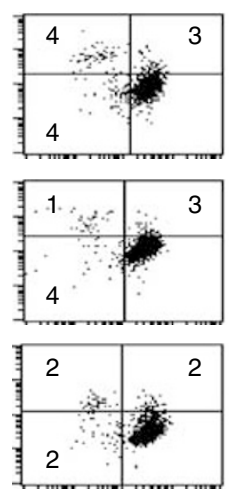

$10^{4} \quad 10^{\circ}$

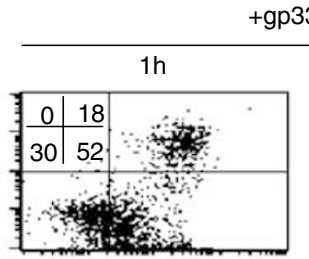

+gp33
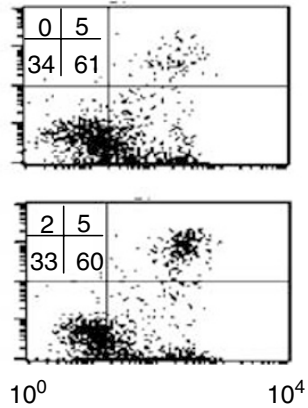

$10^{4} \quad 10^{0}$

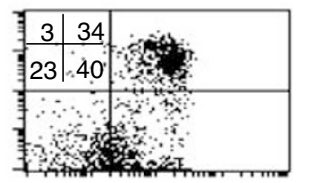

Bid-/-

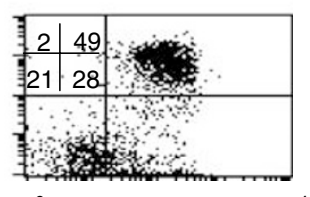

BakxBax-/-

Figure 1 Continued

lines, when assayed at $4 \mathrm{~h}$ of incubation. However, when tested at an earlier time point (i.e. after 1.) a delay in $\Delta \Psi_{\mathrm{m}}$ loss and generation of ROS (Figure 1f), but not of PS exposure and $\mathrm{PI}$ incorporation (Figure 1e) were observed in MEF.Bid ${ }^{-1-}$ and MEF.Bak $\times \mathrm{Bax}^{-1-}$ compared to MEF.wt cells. In relation to MEF.wt cells, up to $90 \%$ of ${\mathrm{MEF} . \mathrm{Bid}^{-/-}}^{-1}$ and MEF.Bak $\times \mathrm{Bax}^{-1-}$ cells died upon incubation with gzmB ${ }^{+}$CTLs for $4 \mathrm{~h}$ at an effector:target (E:T) ratio of 10:1 (Figure 2a). In addition, both MEF.wt and MEF.Bak $\times \mathrm{Bax}^{-1-}$ cells were similarly susceptible to $\mathrm{gzmB}^{+} \mathrm{CTL}$-induced cell death, when tested at different E:T (10:1, 3:1 and 1:1).
In contrast, while the release of cytochrome $c$ was readily detected in MEF.wt, it was almost completely absent in MEF.Bid ${ }^{-1-}$ or MEF.Bak $\times \mathrm{Bak}^{-1-}$ cells (Figure 3a). This process was independent of caspase-3, -7 and -9 as the gzmB $^{+} \mathrm{CTL}-$ mediated release of cytochrome $c$ was reduced neither in MEF.wt cells pretreated with the pan-caspase inhibitor Ac-ZVAD-fmk (Supplementary Figure S4a) nor in MEF.Casp $3 \times 7^{-1-}$ or in MEF.Casp $9^{-1-}$ cells (Figure $3 b$ ). These data indicate that Bid and Bak/Bax are critical for gzmB-induced cytochrome $c$ release, but not for ROS production, suppression of $\Delta \Psi_{\mathrm{m}}$, perturbation of the plasma

Figure $2 \mathrm{gzmB}{ }^{+}$CTLs induce cell death independently of Bid, Bak, Bax and caspase activity. Gp33-pulsed MEF.wt, MEF.Bid ${ }^{-1-}$ and MEF.Bak $\times$ Bax ${ }^{-1-}$ (a) or MEF.wt, MEF.Casp $3 \times 7^{-1-}$ and MEF.Casp $9^{-1-}$ and (b) cells were incubated with ex vivo virus-immune gzmB ${ }^{+}$CTL ( $4 \mathrm{~h}, 10: 1$ effector/target ratio) in the presence or absence of the general caspase inhibitor Ac-ZVAD-fmk ( $80 \mu \mathrm{M})$ or the caspase-3/-7 inhibitor Ac-DEVD-fmk (not shown) (a, $80 \mu \mathrm{M}$ both). Target cell death was monitored by survival assay as described in Materials and Methods. Data are given as mean \pm S.E.M. of five (MEF.wt, MEF.Bid $\left.{ }^{-1-} ; \mathbf{a}\right)$, four (MEF.Bak $\times$ Bax $\left.^{-1-} ; \mathbf{a}\right)$ and three $\left(\right.$ MEF.wt, MEF.Casp $3 \times 7^{-1-}$, MEF.Casp $9^{-1-}$, b) independent experiments, respectively. (c) Gp33-pulsed MEF.wt and MEF.Bak $\times$ Bax $^{-{ }_{-}}$cells were incubated with ex vivo virus-immune gzmB ${ }^{+}$CTL at different effector/target ratio $(4 \mathrm{~h})$ in the presence or absence of the general caspase inhibitor Ac-ZVAD-fmk $(80 \mu \mathrm{M})$. Target cell death was monitored by survival assay as described in Materials and Methods. Representative of two independent experiments. Ctr: only target cells; ctr ZVAD: target cells + ZVAD-fmk 
membrane and, most surprisingly, cell death. Our finding supports some previous work where purified gzmB was used to measure plasma and mitochondrial membrane perturbation, ${ }^{7,21}$ but apparently contradicts those data obtained with Bid-deficient cells where resistance to gzmBmediated cell death was shown. ${ }^{23}$ The role of Bid and a

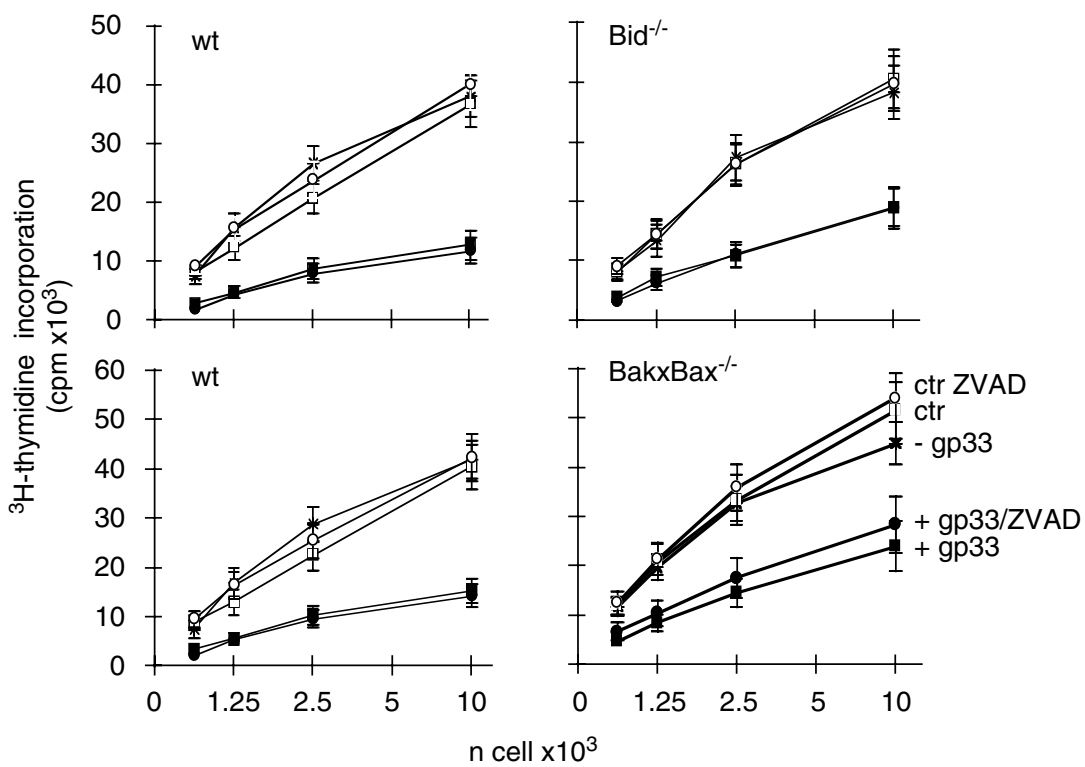

b
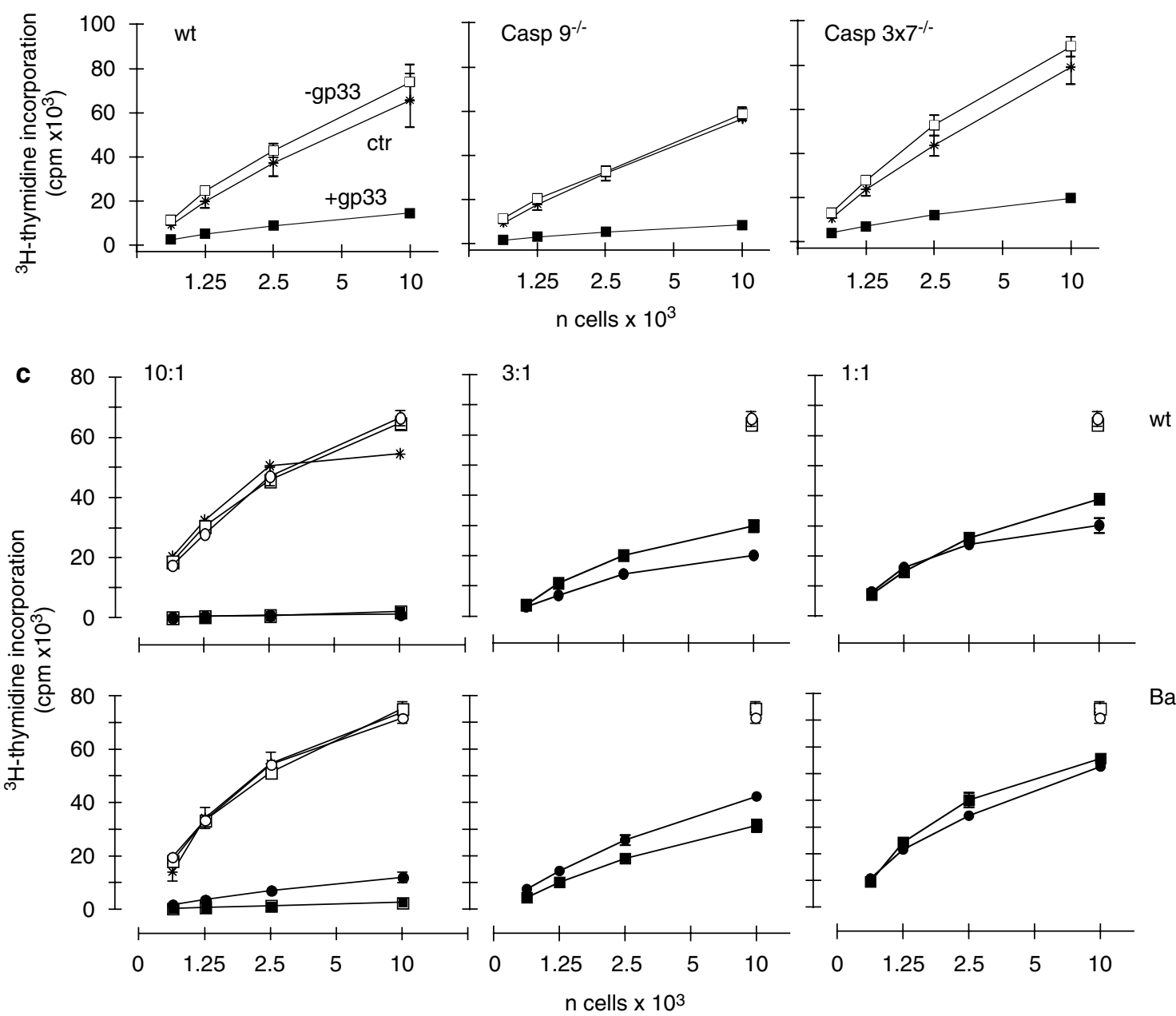

BakxBax $^{-/-}$

$\square \mathrm{ctr}$

O ctr ZVA

* -gp33

- $+\mathrm{gp} 33$

- +gp33/ 
a
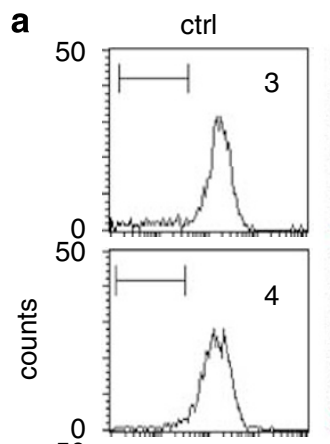

50

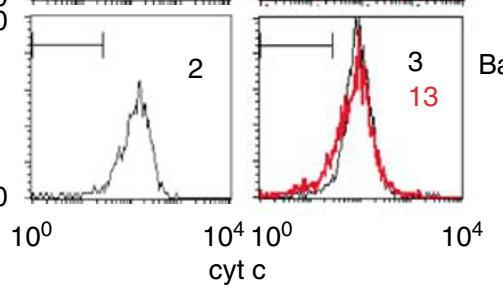

$-/+$ gp 33
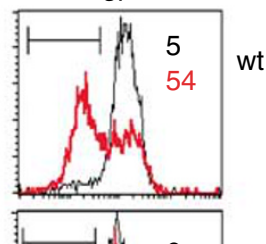

wt

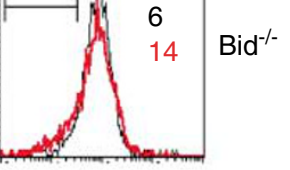

BakxBax ${ }^{-1-}$

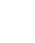

50
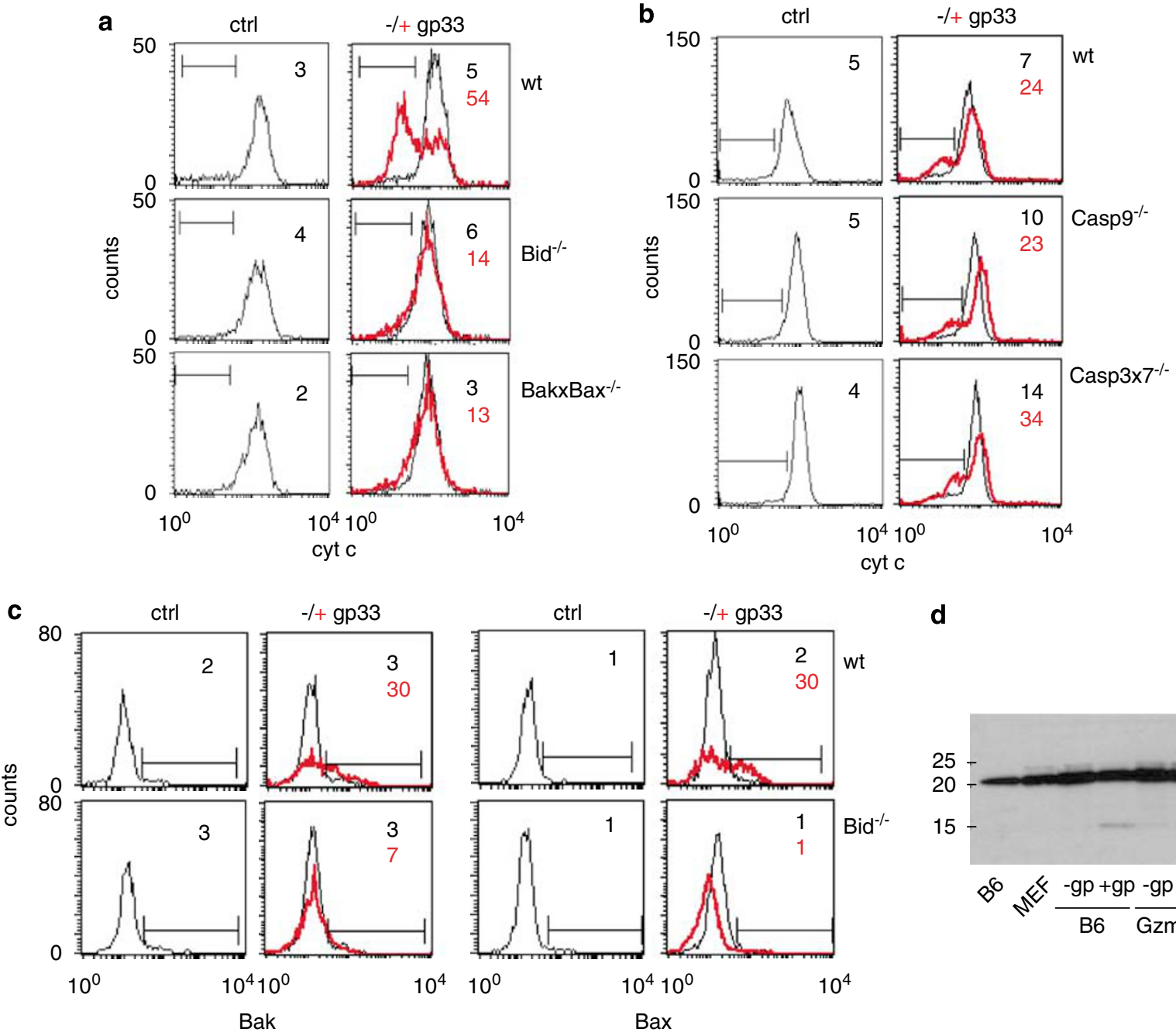

d

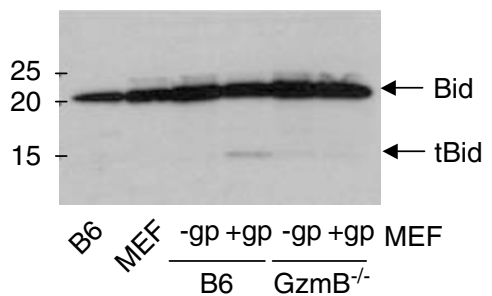

Figure $3 \mathrm{gzmB}{ }^{+}$CTL-induced conformational change of Bak and Bax depends on Bid and is critical for cytochrome $c$ release independent of caspases. Gp33-pulsed MEF.wt (see Materials and Methods), MEF.Bid ${ }^{-1-}$ (a, c) and MEF.Bak $\times \operatorname{Bax}^{-1-}$ (a) or MEF.wt (see Materials and Methods), MEF.Casp $3 \times 7^{-1-}$ and MEF.Casp $9^{-1-}$ (b) cells were incubated with ex vivo virus-immune gzmB ${ }^{+} \mathrm{CTL}(4 \mathrm{~h}, 10: 1$ effector/target ratio). Cytochrome $c$ release from mitochondria (a, $\mathbf{b})$ and Bak and Bax conformational change (c) were analyzed by three-color flow cytometry in the cell population negative for CD8 expression (target cells) as described in Materials and Methods. Histograms show a representative experiment of two independent experiments. Ctr: only target cells. (d) Gp33-pulsed MEF.wt cells were incubated with ex vivo virus-immune B6 or gzmB ${ }^{-1-}$ CTL (1 h, 10:1 effector/target ratio). Subsequently, lysates were prepared and Bid cleavage was analyzed by western blot as described in Materials and Methods. $\mathrm{Bid}=22 \mathrm{kDa}, \mathrm{tBid}=16 \mathrm{kDa}$

Bax/Bak in gzmB-mediated cytochrome $c$ release was further bolstered by the fact that Bid was effectively cleaved in MEF.wt cells after incubation with $\mathrm{gzmB}^{+} \mathrm{CTL}$ but not gzmB ${ }^{-1-}$ CTL (Figure 3d). Moreover, Bax and Bak activation, as determined by the detection of their active state with $\mathrm{N}$-terminal antibodies, was only observed when gzmB ${ }^{+}$CTLs were incubated with MEF.wt, but not with MEF.Bid ${ }^{-1-}$ cells (Figure $3 \mathrm{c}$ ).

Caspase-3 and -7 are activated by gzmB $^{+}$CTL, independently of Bid, Bax and Bak but neither of the two enzymes are required for $\mathrm{gzmB}^{+}$CTL-mediated target cell death. Next, we determined whether effector caspase-3 and -7 were activated and/or required for gzmB ${ }^{+}$CTL-mediated cell death of gp33-pulsed MEFs. For that purpose, $\mathrm{gzmB}^{+} \mathrm{CTL}$ were incubated with MEF.wt cells previously treated with either the general caspase inhibitor
Ac-ZVAD-fmk, the more caspase-3/-7 selective inhibitor Ac-DEVD-fmk or with MEF.Casp $3 \times 7^{-/-}$cells; untreated MEF.wt cells served as a control.

Similar to previous findings with EL4 thymoma cells as targets, ${ }^{6}$ the treatment of MEF.wt cells with Ac-ZVAD-fmk did not prevent target cell death by gzmB ${ }^{+}$CTL (Figure 2a). This was also the case when MEF.Bid ${ }^{-1-}$ and MEF.Bak $\times \mathrm{Bax}^{-1-}$ cells were used as targets under similar conditions (Figure 2a). Furthermore, $\mathrm{gzmB}^{+} \mathrm{CTL}$-mediated target cell death proceeded with similar efficiency in MEF.wt, MEF.Casp $9^{-1-}$ and MEF.Casp $3 \times 7^{-1-}$ (Figure 2b), as well as in MEF.wt and MEF.Bak $\times \mathrm{Bax}^{-1-}$, previously loaded with Ac-ZVAD-fmk, even when lower E:T (3:1 and 1:1) ratios were used (Figure 2c). These data suggest that gzmB-induced target cell death occurs primarily through a caspase-independent mechanism.

To determine if caspase- 3 was nevertheless activated in the gp33-pulsed MEF.wt cells upon $\mathrm{gzmB}^{+} \mathrm{CTL}$ 
a

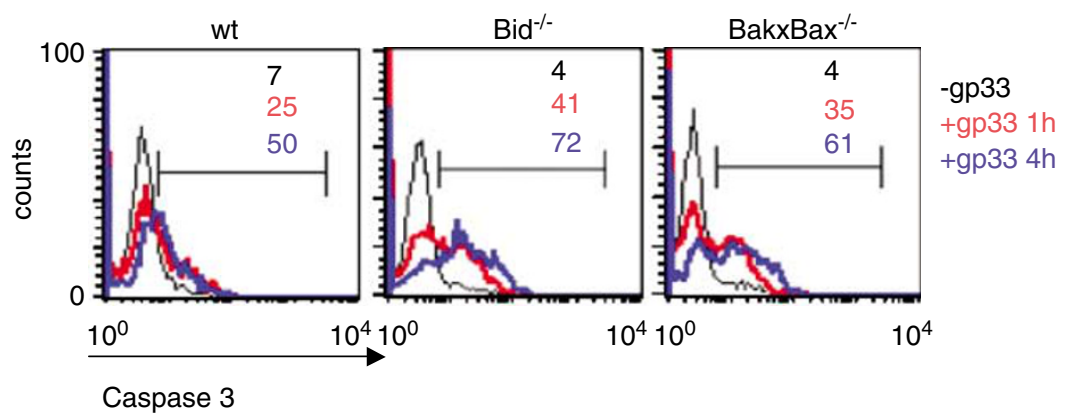

b
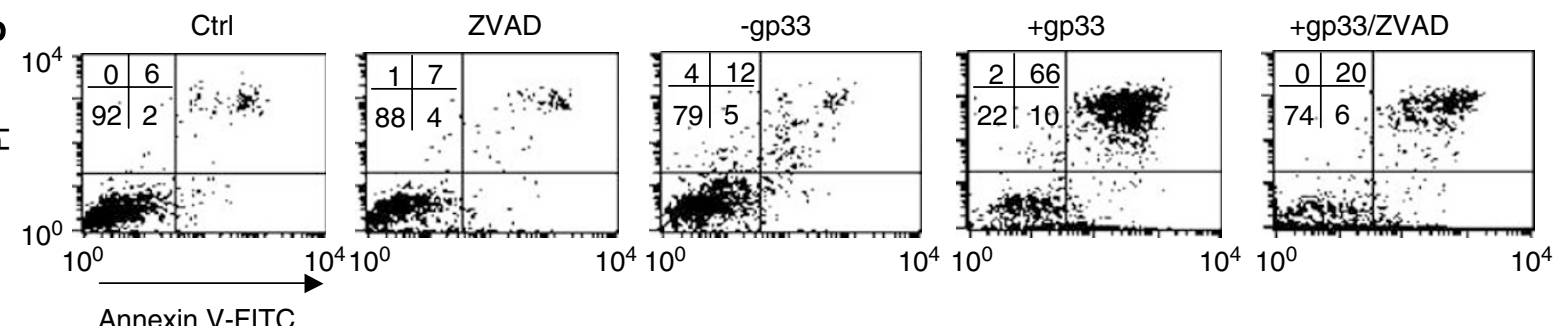

Annexin V-FITC

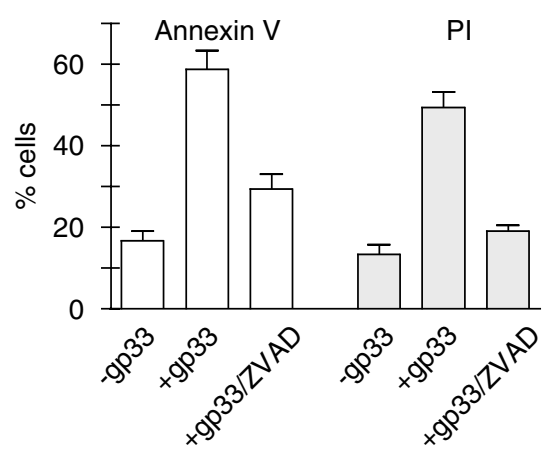

Figure $4 \mathrm{gzmB}{ }^{+}$CTL-induced activation of caspase-3 is independent of Bid or Bak and Bax and is crucial for PS exposure but not for mitochondrial perturbation. (a) Gp33-pulsed MEF.wt, MEF.Bid ${ }^{-1-}$ and MEF.Bak $\times$ Bax $^{-1-}$ were incubated with ex vivo virus-immune gzmB ${ }^{+}$CTL (1 and $4 \mathrm{~h}$ as indicated, 10:1 effector/target ratio). Activation of caspase-3 was monitored with an FITC-labeled mAb against the active form of the enzyme. Histograms show a representative experiment. Numbers correspond to the percentage of cells as indicated by the horizontal bars. (b) gp33-pulsed MEF. wt was incubated with ex vivo virus-immune gzmB ${ }^{+} \mathrm{CTL}(4 \mathrm{~h}, 10: 1$ effector/target ratio) in the presence or absence of the general caspase inhibitor Ac-ZVAD-fmk $(80 \mu \mathrm{M})$. Subsequently, PS exposure on plasma membrane (annexin-V-FITC) and PI uptake (b) were analyzed by three-color flow cytometry in the cell population negative for CD8 expression (target cells) as described in Materials and Methods. A representative experiment is shown in the upper panels. Numbers correspond to the percentage of cells in each quadrant. Data in the graph (PS/PI) are represented as mean \pm S.E.M. of three independent experiments. Ctr: only target cells; ZVAD: target cells + ZVAD-fmk

co-incubation, we measured caspase-3 activation by FACS analysis using an antibody against the active form of caspase3 (mAb C92-605). Figure $4 a$ shows that about 25 and $50 \%$ of the MEF.wt cells exhibited increased caspase- 3 activation upon $\mathrm{gzmB}^{+} \mathrm{CTL}$ challenge in the presence of the gp33 peptide for 1 and $4 \mathrm{~h}$, respectively. Comparable numbers of cells expressing active caspase- 3 were found in $\mathrm{gzmB}^{+} \mathrm{CTL}$ treated MEF.Bid ${ }^{-1-}(41 \%, 1 \mathrm{~h} ; 72 \%, 4 \mathrm{~h})$ and MEF.Bax/Bak ${ }^{-/}$ $(35 \%, 1 \mathrm{~h} ; 61 \%, 4 \mathrm{~h})$ (Figure $4 \mathrm{a})$, indicating that gzmB most likely cleaved and activated caspase-3 directly, bypassing the need of mitochondrial events such as Bid-induced Bax/Bak activation and cytochrome $c$ release.

gzmB ${ }^{+}$CTL-induced activation of caspase- 3 and -7 is needed for PS surface exposure and plasma membrane permeability. The question remained for what purpose, if not cell death, caspase-3 was activated in the gp33-pulsed target cells in response to $\mathrm{gzmB}^{+} \mathrm{CTL}$ incubation. We therefore first tested the contribution of caspases in general and caspase- 3 and -7 in particular in gzmB ${ }^{+}$CTL-induced PS exposure and plasma membrane permeability. Figure $4 \mathrm{~b}$ shows that when Ac-ZVAD-fmk was added to gp33-pulsed MEF.wt cells incubated with gzmB ${ }^{+} \mathrm{CTL}$, the percentages of annexin- $\mathrm{V}^{+}$and $\mathrm{PI}^{+}$target cells were drastically reduced as compared to untreated cells as shown previously. ${ }^{6}$ A similar result was obtained with $\mathrm{gzmB}^{+}$CTL-treated MEF.Casp $3 \times 7^{-1-}$ cells, while MEF.wt cells showed a marked increase in PS exposure and PI permeability (Figure 5a). Using MEF.Casp $3^{-1-}$ and MEF.Casp $7^{-1-}$ cells to analyze the individual roles of caspase- 3 and -7 in this process, we noted a synergistic effect of caspase- 3 and -7 (Figure $5 c$ ); remarkably, caspase- 7 was able to replace caspase- 3 in inducing membrane damage. In contrast, caspase-9, a caspase-3-activating initiator caspase of the mitochondrial pathway, did not play any role in these effects as MEF.Casp $9^{-1-}$ cells were indistinguishable from MEF.wt 


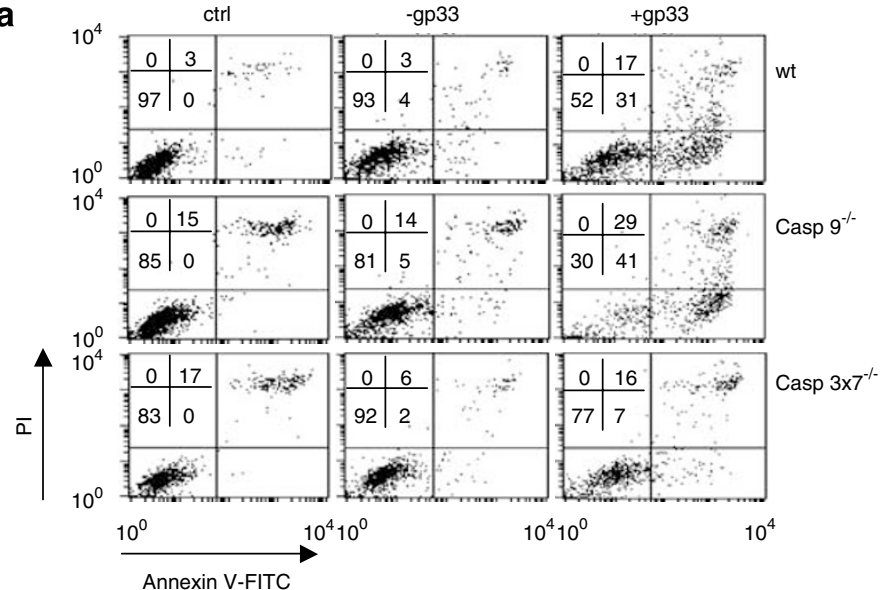

b

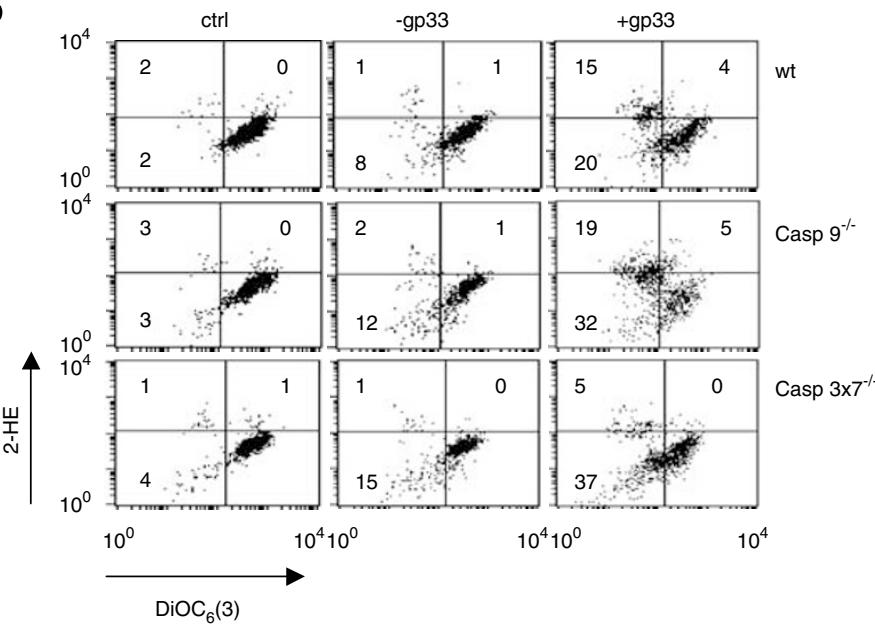

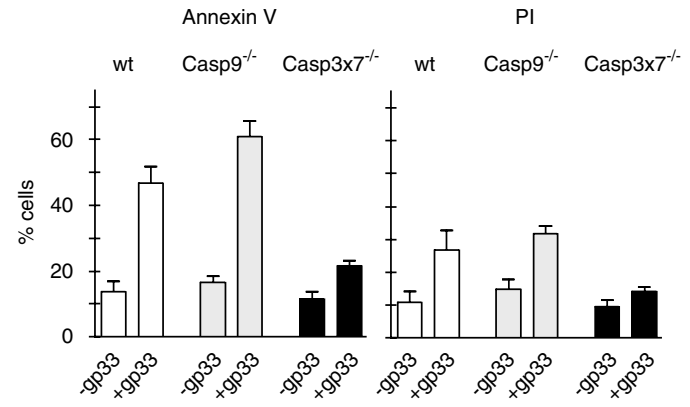

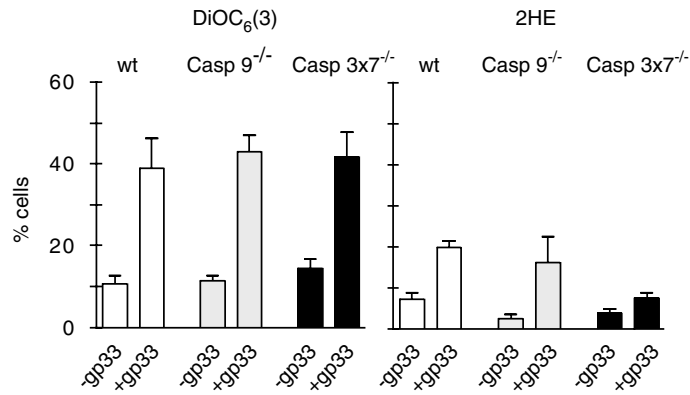

C

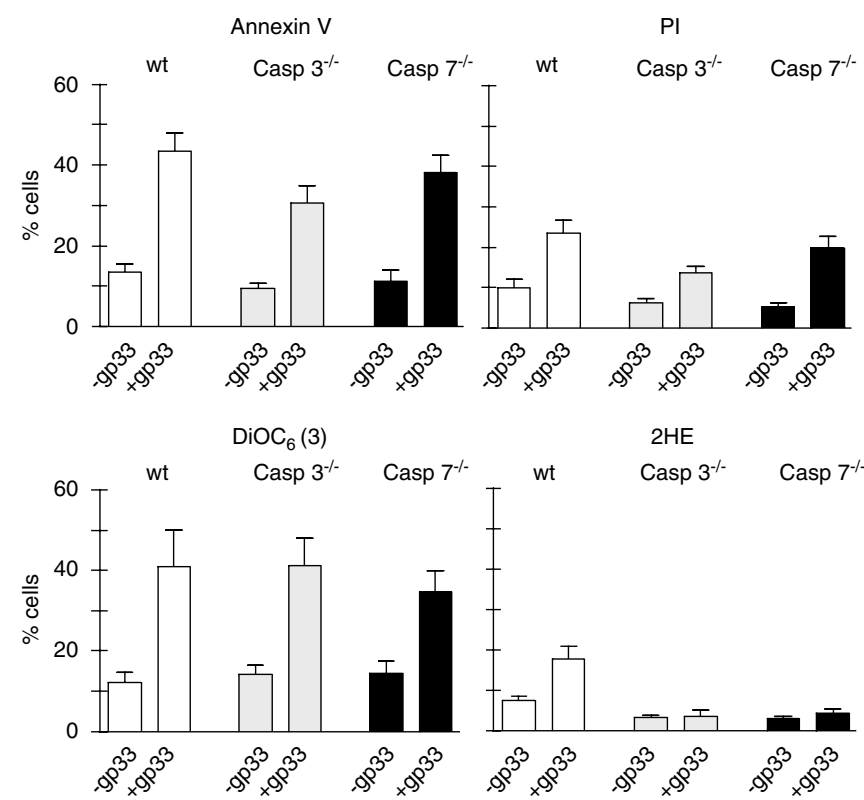

Figure 5 Role of caspase-3, -7 and -9 in gzmB ${ }^{+}$CTL-induced membrane and mitochondrial perturbation. Gp33-pulsed MEF.wt, MEF.Casp $3 \times 7^{-1-}$ and MEF.Casp $9^{-1-}$ $(\mathbf{a}, \mathbf{b})$ or MEF.wt, MEF.Casp $3^{-1-}$ and MEF.Casp $7^{-1-}$ (c) cells were incubated with ex vivo virus-immune gzmB ${ }^{+} \mathrm{CTL}$ (4h, 10:1 effector/target ratio). Subsequently, PS exposure and PI uptake $(\mathbf{a}, \mathbf{c})$ and, in parallel, suppression of $\Delta \Psi_{\mathrm{m}}$ and ROS generation $(\mathbf{b}, \mathbf{c})$ were analyzed by three-color flow cytometry in the cell population negative for CD8 expression as described in Materials and Methods. (a, b) A representative experiment is shown in the left panels. Numbers correspond to the percentage of cells in each quadrant. Data in the graphs (a, b, right panels) are represented as mean \pm S.E.M. of five (MEF.wt, MEF.Casp $3 \times 7^{-l-}$ ) and three (MEF.Casp $9^{-l-}$ ) independent experiments, respectively. (c) Data in the graphs are represented as mean \pm S.E.M. of five (MEF.wt, MEF.Casp $3^{-1-}$ ) and three (MEF.Casp $7^{-l-}$ ) independent experiments, respectively. Ctr: only target cells 
cells in gzmB-induced annexin- $V$ expression and $\mathrm{PI}$ incorporation (Figure 5a). These data extend previous findings by us and others showing that caspase- 3 activation results in surface exposure of PS and increased plasma membrane permeability. ${ }^{6,8,12}$ However, these results suggest that $\mathrm{gzmB}^{+} \mathrm{CTL}$-induced PS exposure on target cells does not depend on mitochondrial signaling (Bax/Bak activation, cytochrome $c$ release, caspase- 9 activation), at least at early stages of CTL-target cell interaction $(4 \mathrm{~h})$, and are not required for final $\mathrm{gzmB}^{+} \mathrm{CTL}$-induced target cell killing (see above, Figure $2 \mathrm{a}$ and $\mathrm{b}$ ).

gzmB ${ }^{+}$CTL-induced activation of caspase-3 and -7 but not caspase- 9 is needed for ROS production, but not for the loss of the mitochondrial membrane potential. We next tested the role of caspase-3, -7 and -9 in gzmB $^{+}$CTLinduced $\Delta \Psi_{\mathrm{m}}$ loss and the generation of ROS in MEF.wt, MEF.Casp $3 \times 7^{-1-}$ and MEF.Casp $9^{-1-}$ cells (Figure $5 b$ ). We found that $\mathrm{gzmB}^{+} \mathrm{CTL}$ induced comparable levels of $\Delta \Psi_{\mathrm{m}}$ loss in MEF.wt, MEF.Casp $3 \times 7^{-1-}$ and MEF.Casp $9^{-1-}$ cells (Figure $5 \mathrm{~b}$ ). This indicates that gzmB-induced reduction of $\Delta \Psi_{\mathrm{m}}$ is independent of direct or indirect - via caspase-9 and apoptosome formation - activation of effector caspases. In contrast, ROS generation was significantly reduced in MEF.Casp $3 \times 7^{-1-}$ as compared to MEF.wt cells (Figure 5b). Both caspases seem to be needed for optimal induction of ROS, as MEF.Casp $3^{-1-}$ and MEF.Casp $7^{-1-}$ cells showed similar impairment in ROS production as MEF.Casp $3 \times 7^{-1-}$ cells (Figure $5 b$ and $c$ ). These data imply that gzmB-induced ROS generation critically depends on the activation of effector caspases, in particular caspase-3 and -7. However, as for PS exposure and PI uptake, ROS production was probably the result of a direct activation of caspase-3 and -7 by gzmB (bypassing mitochondria) because MEF.Casp $9^{-1-}$ (Figure $5 b$ ) as well as MEF.Bid ${ }^{-1-}$ and MEF.Bax/Bak ${ }^{-1-}$ MEF (Figure $1 b$ and d) cells exhibited similar ROS production in response to $\mathrm{gzmB}^{+} \mathrm{CTL}$ as MEF.wt cells.

gzmB ${ }^{+}$CTL-induced loss of the mitochondrial membrane potential is triggered via two independent pathways. In Figures 1 and 5, we show that MEF.Bid ${ }^{-1-}$ and MEF.Bak $\times \mathrm{Bax}^{-1-}$ as well as MEF.Casp $3 \times 7^{-1-}$ exhibit a similar loss of $\Delta \Psi_{\mathrm{m}}$ as seen in MEF.wt cells, indicating that this pro-apoptotic response was neither individually dependent on Bid/Bax/Bak nor on caspase-3/-7. However, it is possible that once Bid or Bax/Bak are deleted, the loss of $\Delta \Psi_{\mathrm{m}}$ is mediated via caspases. As shown in Figure 6 , this was indeed the case. When gzmB ${ }^{+}$CTLs were incubated with gp33-pulsed MEF.Bid ${ }^{-1-}$ and MEF.Bak $\times \mathrm{Bax}^{-1-}$ in the presence of the general caspase inhibitor Ac-ZVAD-fmk or the caspase-3/-7 inhibitor Ac-DEVD-fmk, the induction of $\Delta \Psi_{\mathrm{m}}$ loss was severely diminished (Figure 6). Together with the finding that the levels of $\Delta \Psi_{\mathrm{m}}$ loss and ROS production were diminished in MEF.Bid ${ }^{-1-}$ and MEF.Bak $\times \mathrm{Bax}^{-1-}$ compared to MEF.wt, when incubated with gzmB ${ }^{+} \mathrm{CTL}$ for $1 \mathrm{~h}$, but not $4 \mathrm{~h}$ (Figure $1 \mathrm{f}$ ), the data indicate that gzmB is able to induce two independent pathways of mitochondrial depolarization: one mediated by Bid-dependent Bak/Bax
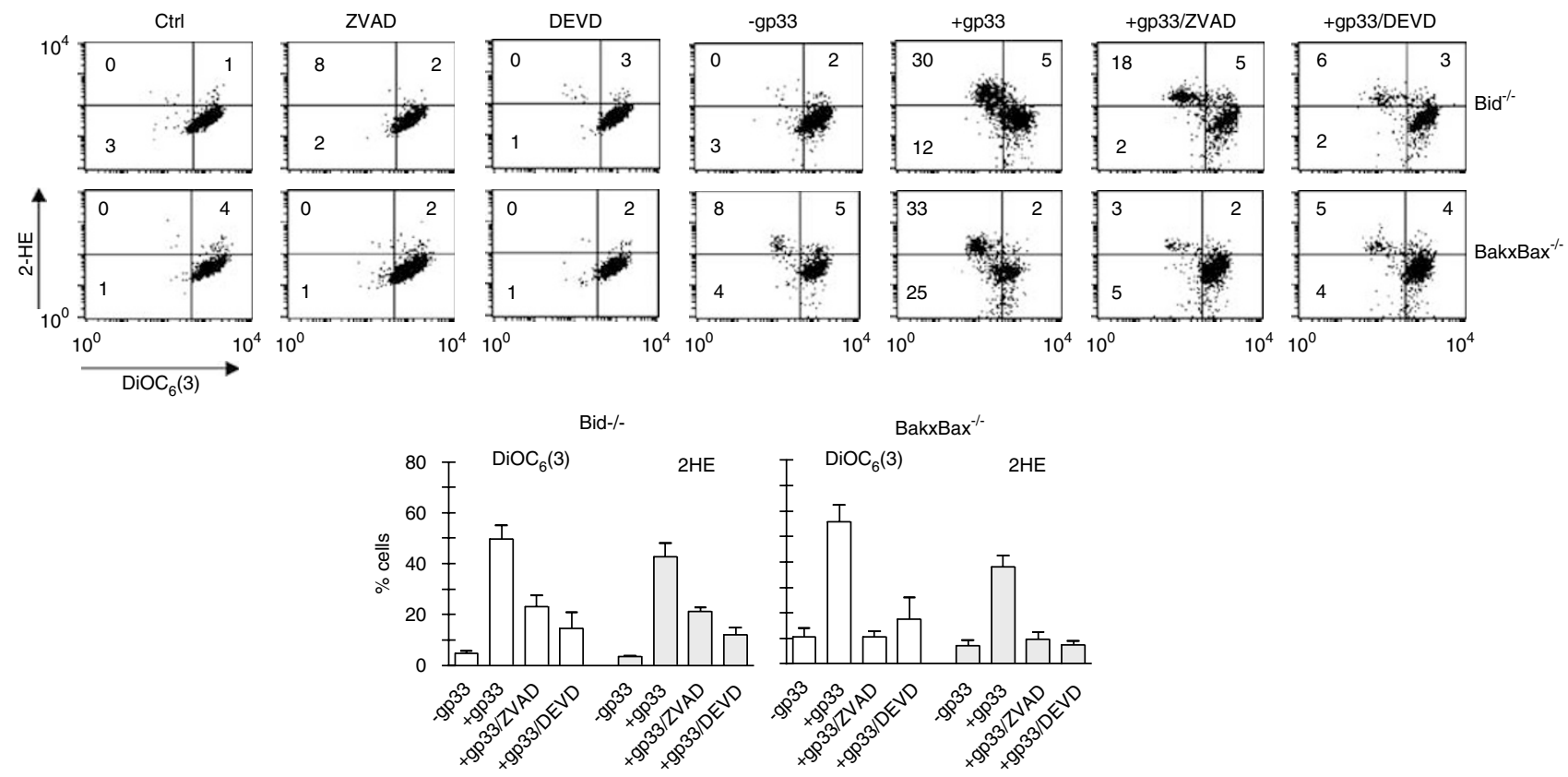

Figure $6 \mathrm{gzmB}^{+} \mathrm{CTL}$-induced mitochondrial damage is blocked in the absence of Bak and Bax and simultaneous inhibition of caspase-3 and -7. Gp33-pulsed MEF.Bid ${ }^{-1-}$ and MEF.Bak $\times \mathrm{Bax}^{-1-}$ cells were incubated with ex vivo virus-immune gzmB ${ }^{+} \mathrm{CTL}(4 \mathrm{~h}, 10: 1$ effector/target ratio) in the presence or absence of the general caspase inhibitor Ac-ZVAD-fmk or the caspase-3/-7 inhibitor Ac-DEVD-fmk ( $80 \mu \mathrm{M}$ both). $\Delta \Psi_{\mathrm{m}}$ loss and ROS generation were analyzed by three-color flow cytometry in the cell population negative for CD8 expression (target cells) as described in Materials and Methods. A representative experiment is shown in the upper panels. Numbers correspond to the percentage of cells in each quadrant. Data in the graphs are represented as mean \pm S.E.M. of three (ZVAD) and two (DEVD) independent experiments, respectively. Ctr: only target cells; ZVAD: target cells + ZVAD-fmk; DEVD: target cells + ZVAD-fmk 
activation in the absence of caspase (Figure $5 b$ ) and the other by activation of both caspase-3 and -7, and independent of $\mathrm{Bid} / \mathrm{Bak} / \mathrm{Bax}$ (Figure $\mathrm{b}$ and $\mathrm{d}$ ). Only when both pathways are simultaneously inhibited, then the integrity of mitochondria from gzmB-challenged target cells is maintained. However, gzmB ${ }^{+} \mathrm{CTL}-$ mediated cell death still occurs under the latter conditions, as revealed by a cell survival assay (Figure 2a).

\section{Discussion}

The multitude of independent and/or interconnecting proapoptotic pathways, which have been described so far renders it difficult to define a general model of gzmB-induced cell death. This may in part be due to the fact that gzmB proteins, CTLs and target cells from different species have been used interchangeably, but they in fact exhibit distinct qualities. ${ }^{24-26}$ In addition, the substrate specificity of gzmB in cell-free systems may be different from those of intact cells.

The present study was designed to analyze pro-apoptotic processes induced by native mouse gzmB under physiological conditions, by using ex vivo-derived, virus-immune mouse gzmB ${ }^{+}$CTL as a source of gzmB, wt and mutant MEFs as target cells and low effector to target cell ratios $(E: T=10: 1)$. The data show that gzmB induces plasma membrane damage by a caspase-3 or -7-dependent pathway, but independently of mitochondrial signaling (Figure 7). The same is true for gzmB-mediated ROS production. However, gzmB induces

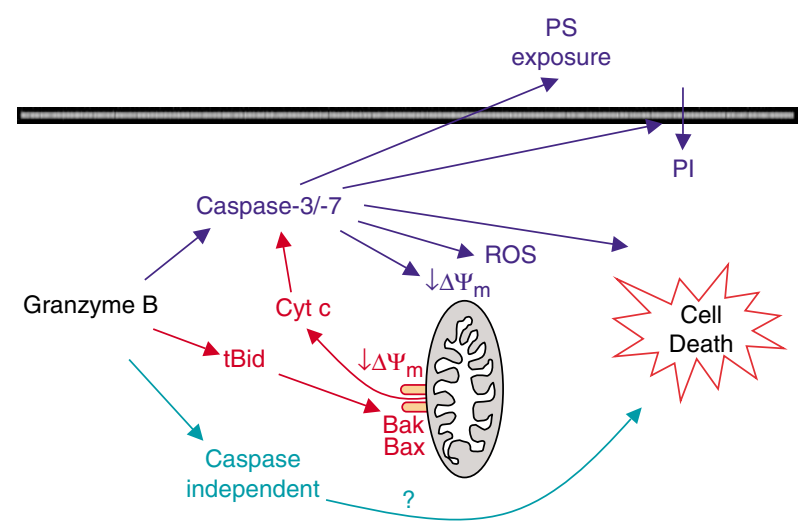

Figure 7 Model for gzmB-mediated pro-apoptotic pathways and cell death elicited by ex vivo CTL. gzmB exerts pleiotropic pro-apoptotic potential in gzmB $^{+}$CTL-induced cell death. First, gzmB directly activates caspase-3 and -7 , independently of mitochondrial events such as Bid-induced Bak/Bax activation and cytochrome $c$ release. Caspase-7 can replace caspase-3 in the induction of plasma membrane perturbance (PS exposure and PI permeability), and both caspases are critical in gzmB-mediated ROS production. However, both processes are dispensable for gzmB ${ }^{+}$CTL-mediated cell death. Second, gzmB-induced activation of Bid and Bak/Bax is independent of caspase- 3 and -7 and leads to cytochrome $c$ release. The absence of Bid or Bak/Bax does not interfere with gzmB ${ }^{+} \mathrm{CTL}$ induced cell death. Third, gzmB-mediated mitochondrial perturbation is triggered by two distinct pathways, one involving Bid/Bax/Bak, independent of caspases, to induce $\Delta \Psi_{\mathrm{m}}$ loss and cytochrome $c$, and one triggering caspase-3/-7 activation without the implication of Bid/Bax/Bak to induce $\Delta \Psi_{\mathrm{m}}$ loss and ROS production. Only when both pathways are simultaneously blocked, mitochondrial depolarization is prevented. Finally, gzmB kills target cells independent of caspases and mitochondrial signals, including PS exposure, mitochondrial depolarization, ROS production and cytochrome $c$ release mitochondrial perturbation by two distinct processes, one involving caspase-3 and/or -7 independently of Bax/Bak to trigger loss of $\Delta \Psi_{m}$ and ROS production, and the other engages Bid and Bak $\times$ Bax in the absence of caspase activation to provoke loss of $\Delta \Psi_{\mathrm{m}}$ and cytochrome $c$ release (Figure 7). The additional finding that $\mathrm{gzmB}^{+} \mathrm{CTL}$ abrogates target cell proliferation, most likely by inducing cell death, even upon inhibition of both, caspases and mitochondrial events, suggests additional cytosolic targets that mediate gzmB-induced cell death and emphasizes the pleiotropic proapoptotic potential of gzmB.

Genetic and functional analyses indicate that gzmB is the only protease contributing to the gzmB ${ }^{+} \mathrm{CTL}-$ mediated proapoptotic and cytolytic activities observed. Although all ex vivo-derived virus-immune CTLs expressed transcripts for gzmK the putative protein did not act by itself, as $\mathrm{gzmA} \times \mathrm{B}^{-/-}$ CTL, which expressed perf and gzmK transcripts, but neither gzmA, gzmB or any of the other gzms, including C, D, E, F and $\mathrm{M}$, were able to induce any of the pro-apoptotic processes analyzed or to cause cell death under similar conditions (Supplementary Figure S1d; 3a-c; and Pardo and colleagues $^{6,27}$ and Revell et al. $\left.{ }^{28}\right)$. This contrasts with a recent report which suggests that $\mathrm{gzmA} \times \mathrm{B}^{-1-} \mathrm{CTLs}$ are able to induce, at least to some limited extent, cell death and proapoptotic processes, such as PS translocation, by mechanisms distinct from classical apoptosis. ${ }^{29}$ This discrepancy may be due to the fact that the latter studies employed in vitropropagated rather than ex vivo CTL, and that in vitrocultivated CTLs express different patterns of gzms, including gzmC (Revell et al. ${ }^{28}$ and own unpublished results), distinct from those seen in ex vivo-derived LCMV-immune gzmB ${ }^{+} \mathrm{CTL}$. The data obtained with ex vivo-derived LCMVimmune perf ${ }^{-1-}$ and gzmA $\times \mathrm{B}^{-1-} \mathrm{CTL}$ also exclude a contribution of the FasL/Fas system to $\mathrm{gzmB}^{+} \mathrm{CTL}$-mediated pro-apoptotic processes, including cell death, of MEFs observed.

The notion that caspases, in particular caspase-3, are critical for gzmB-induced plasma membrane disturbance ${ }^{6,8}$ is substantiated by our finding showing that gzmB ${ }^{+}$CTL did not induce PS exposure in caspases-3/-7-deficient MEFs. In addition, we show for the first time that caspase- 7 can replace caspase- 3 in this gzmB-mediated process and that both enzymes synergize in the pro-apoptotic program. The fact that gzmB induced similar levels of PS exposure in MEF.wt, MEF.Bid ${ }^{-1-}$, MEF.Bak $\times \mathrm{Bax}^{-1-}$ and MEF.Casp $9^{-1-}$ cells, also suggests that activation of caspase-7, like caspase- $3,^{13}$ is an early event in target cell apoptosis and independent of mitochondrial events, including cytochrome $c$ release and subsequent caspase- 9 activation. In light of a recent report showing that caspase-7 is more sensitive to proteolysis by gzmB compared to caspase- $3,{ }^{13}$ we postulate an important role for caspase-7 in the early phase of CTL-mediated membrane disturbance via gzmB. Although the putative caspase-7 substrate(s), critical for this process, has not yet been identified it is possible that caspase-7, like caspase-3, contributes to plasma membrane blebbing by cleaving the actin remodeling protein gelsolin. ${ }^{30,31}$ Alternatively, caspase7 or other caspases may synergize with gzmB in targeting and perturbing the cytoskeleton by cleaving $\alpha$-tubulin, the tubulin folding cofactor and/or other relevant proteins, such as filamin and ROCKII. ${ }^{14,15,32}$ However, the data do not exclude the 
possibility that gzmB ${ }^{+}$CTL-induced and caspases-3/-7-facilitated PS exposure is modulated by additional processes, including mitochondrial events, under different conditions. Different CTL population may express or deliver different amounts of gzmB causing target cells to enter distinct pathways of apoptosis.

The observation that gzmB ${ }^{+} \mathrm{CTL}$ induced ROS production in MEF.wt, MEF.Bid ${ }^{-1-}$ and MEF.Bak $\times \mathrm{Bax}^{-1-}$ cells, but not in MEFs deficient in caspase-3, -7 or both, is novel. It suggests that a concerted action of the two caspases is required in ROS production and independent of Bax/Bak, but not necessarily caspase-mediated mitochondrial membrane perforation. This extends previous findings which showed that caspase- 3 induces ROS production by targeting the p75 subunit of respiratory chain complexes I and II in previously permeabilized mitochondria. ${ }^{9}$ However, our data are at variance with a recent report showing that purified rat gzmB triggered ROS production from isolated mouse mitochondria, even in the absence of the cytosolic $\mathrm{S} 100$ fraction. ${ }^{20}$ Although unclear at present, this discrepancy may be due to the fact that gzmB and mitochondria were derived from different species. The combined data, however, indicate that mouse gzmB, when delivered via $C T L$ is unable to directly target mitochondria and reveal caspase- 3 and -7 as the main executors during gzmB $^{+}$CTL-induced ROS production.

Our data do not discriminate whether gzmB ${ }^{+} \mathrm{CTL}$-induced ROS only derives from mitochondria, in particular by interference of caspase-3 with the electron transport chain, as suggested before ${ }^{9}$ or from other sources, such as peroxisomes, the cytosolic NADPH oxidases or cytochrome $\mathrm{P}-450$ enzymes in the endoplasmic reticulum. Future studies will have to identify the exact intracellular source of ROS during gzmB-dependent target cell killing.

gzmB ${ }^{+}$CTL similarly induced $\Delta \Psi_{\mathrm{m}}$ loss in both MEF.Bid ${ }^{-1-}$ and MEF.Bak $\times \mathrm{Bax}^{-1-}$, and in MEF.Casp $3 \times 7^{-1-}$ cells, indicating that gzmB triggers two independent pathways resulting in mitochondrial depolarization. These data corroborate, at least partially, previous studies ${ }^{7,8,17,21}$ and suggest that gzmB elicits $\Delta \Psi_{\mathrm{m}}$ loss either via Bid cleavage and subsequent activation of Bak/Bax or by engaging caspase-3 and -7 .

The pro-apoptotic events, which accompany the loss of $\Delta \Psi_{\mathrm{m}}$ by these two pathways, are however distinct: whereas the $\mathrm{Bid} / \mathrm{Bad} / \mathrm{Bak}$-dependent pathway involves cytochrome $c$ release, the caspase-3/-7-dependent one is accompanied by ROS production. Interestingly, both pathways appear to be redundant for $\mathrm{gzmB}^{+} \mathrm{CTL}$-induced apoptosis, since both have to be blocked to rescue cells from $\Delta \Psi_{\mathrm{m}}$ loss. The additional finding that $\mathrm{gzmB}^{+} \mathrm{CTL}$ did not induce $\Delta \Psi_{\mathrm{m}}$ loss and ROS generation in MEF.Bid ${ }^{-1-}$ or MEF.Bak $\times \mathrm{Bax}^{-1-}$ cells, whose caspases were inhibited by Ac-ZVAD-fmk or Ac-DEVD-fmk, excludes the possibility that gzmB modulates $\Delta \Psi_{\mathrm{m}}$ by other, yet unidentified cytosolic and/or mitochondrial proteins as previously suggested by using recombinant mouse gzmB and MEF cells. ${ }^{7}$ Although this discrepancy has not been resolved, two reasons may be responsible for it; the use of purified gzmB versus ex vivo CTLs and/or the effect of additional factors in the LAK extracts used, as the perf source to deliver gzmB. ${ }^{7}$

gzmB $^{+} \mathrm{CTL}$-induced cytochrome $c$ release was absent in Bid- or Bak $\times$ Bax-deficient MEFs, but proceeded normally in cells lacking caspase- 9 or caspase-3, -7 or both. This finding supports previous studies showing that gzmB induces mitochondrial outer membrane permeabilization, via direct cleavage of Bid instead of activating caspase-3, leading to Bak/Bax activation and subsequent release of pro-apoptotic proteins such as cytochrome $c$ from the mitochondrial intermembrane space. ${ }^{8,12}$ An alternative pathway has been proposed by which gzmB cleaves the survival factor $\mathrm{Mcl}-1$ to release its $\mathrm{BH} 3$-only partner $\mathrm{Bim}^{33}$ with subsequent activation of Bak/Bax. The strict dependence of cytochrome $c$ on Bid in our study excludes, however, a role for Bim as Bak/Bax activator in this $\mathrm{gzmB}^{+} \mathrm{CTL}-$ mediated process.

The relationship between gzmB-mediated activation of caspases and mitochondrial events, in particular the differential interdependence of Bid and caspase-3 in these processes, has been a matter of controversy. ${ }^{17,21}$ Our results obtained with a physiologically relevant gzmB ${ }^{+} \mathrm{CTL}$-target cell system clearly demonstrate that gzmB can do both, readily induce caspase-3/-7 activation and subsequently cause plasma membrane perturbation and ROS production independently of Bid, Bax or Bak (in MEF.Bid ${ }^{-1-}$ and MEF.Bak $\times \mathrm{Bax}^{-1-}$ cells) as well as use Bid cleavage and Bax/Bak activation to induce cytochrome $c$ release in the absence of caspase-3/-7 activation (in MEF.Casp $3 \times 7^{-1-}$ cells). The latter finding supports data by some $e^{7,8,17}$ but contradicts other studies, which showed that cytochrome $c$ release by gzmB was inhibited by the caspase inhibitor Z-VAD-fmk. ${ }^{34,35}$

The most novel finding of our study was that gzmB ${ }^{+}$CTLabrogated target cell proliferation, most probably by inducing cell death, as monitored by cell survival assays, occurs in the absence of mitochondrial damage (i.e. Bax/Bak activation) and activation of caspases. This result is consistent with and extends our previous study showing that $\mathrm{gzmB}^{+} \mathrm{CTL}-$ mediated cell death could neither be inhibited by the general caspase inhibitor Ac-ZVAD-fmk nor the antioxidant agent NAC. ${ }^{6}$ Whether this type of gzmB-induced abrogation of cell proliferation or cell death is characterized by apoptotic or necrotic features, or by both, and whether this pathway of cell arrest or death is of advantage in host defense against pathogens is not yet known and requires further experimentation. However, the fact that one of the early pro-apoptotic feature, i.e. plasma membrane disturbance, including PS exposure, is not observed in target cells that die independently of caspases suggests that they are refractory to elimination by phagocytosis via the PS receptor ${ }^{11}$ and may hence induce pathological processes in vivo.

The finite genetic space available to the host for its immune defense requires enormous plasticity in its effector function to encompass all the evasion strategies of the multitude of pathogens. The immune system has achieved this in its recognition phase by generating diversity by genetic means of recombination and somatic mutation. Effector mechanisms, such as cytotoxicity leading to cell death via apoptosis, on the other hand have greater constraints in expressing plasticity. One way in possibly achieving diversity of effector function is by generating multiple distinct death pathways employing a limited number of effector molecules.

The data presented here provide evidence that mouse gzmB, one of the important effector molecules of CTL and 
NK cells, can function via a multitude of pathways all leading to cell death. This plasticity is most likely the result of evolutionary pressures from pathogens trying to evade immune elimination, including cellular cytotoxicity. One example being poxvirus-encoded serine protease inhibitors that can severely curtail cytolytic effector functions, most probably by inhibiting Fas-mediated and caspase-facilitated PS exposure, resulting in uncontrolled viral spread. ${ }^{36}$ This diversity in effector function of cytolytic pathways will also be to the host's benefit in tumor elimination.

Finally, our data provide evidence that conclusions drawn from experimental results employing more reductionist approaches (i.e. using recombinant enzymes in artificial in vitro settings) may be erroneous in biological context.

\section{Materials and Methods \\ Mouse strains. Inbred B6, and mouse strains deficient for perf (perf ${ }^{-1-}$ ), gzmA $\left(\mathrm{gzmA}^{-1-}\right)$ and $\mathrm{gzmA} \times \mathrm{B}\left(\mathrm{gzmA} \times \mathrm{B}^{-1-}\right)$, bred on the $\mathrm{B} 6$ background were maintained at the Max-Planck-Institut für Immunbiologie, Freiburg and analyzed for their genotypes as described. ${ }^{6}$ Male mice of 8-10 weeks of age were used in all experiments and were performed in accordance with the local animal care commission.}

Generation of ex vivo CD8 ${ }^{+}$cells. Mice were infected with $10^{5}$ p.f.u. LCMVWE i.p. according to established protocols. ${ }^{6}$ On day 8 postinfection, $\mathrm{CD}^{+}$cells were positively selected from spleen using $\alpha$-CD8-MicroBeads (Miltenyi Biotec, Bergisch Gladbach, Germany) with an autoMACS (Miltenyi Biotec) and resuspended in MEM $/ 5 \%$ FCS prior to use in cytotoxic assays. Purity of selected CD8 ${ }^{+}$cells was assessed by FACS staining and found to be between 95 and $98 \%$.

Cell lines, cell culture and reagents. SV40-transformed MEFs were cultured in MEM supplemented with $10 \% \mathrm{FCS}$ and 2-mercaptoethanol $\left(10^{-5} \mathrm{M}\right)$ at $37^{\circ} \mathrm{C}, 7 \% \mathrm{CO}_{2}$. $\mathrm{Bid}^{-1-}$ and $\mathrm{Bax}^{-1-} \times \mathrm{Bak}^{-1-} \mathrm{MEFs}$ were generated and generously provided by the SJ Korsmeyer laboratory and compared to an MEF.wt cell line, generated by the same group. Casp $9^{-1-}$, Casp $3^{-1-}$, Casp $7^{-1-}$ and Casp $3 \times 7^{-1-}$ MEFs were generated by the group of Richard A Flavell ${ }^{22}$ and compared to an MEF.wt cell line generated by the same group. The genotype of the various MEF cells was periodically tested by genomic PCR and western blot analysis. In some cases, the general caspase inhibitor Ac-ZVAD-fmk or the caspase-3/-7 inhibitor Ac-DEVD-fmk (Bachem; $100 \mu \mathrm{M}$ both) were added to cell cultures as described. ${ }^{6}$ The mouse cell lines 1.3E6 (CTL) and EL4.F15 (EL4) were cultured as described. ${ }^{37}$

RT-PCR. Total RNA was extracted from up to $5 \times 10^{6}$ cells, using the QIAshredder spin columns, the RNeasy Mini Kit and the RNase-free DNase Kit (all from Qiagen, Hilden, Germany) according to the manufacturer's instructions, and specific transcripts were amplified. The sense/antisense primers for $g z m A$ and Hprt1 and gzmC, gzmD, gzmE, gzmF and gzmG are described in Martin et al. ${ }^{37}$ and Revell et al., ${ }^{28}$ respectively. Sense/antisense primers for $g z m B, g z m K$ and gzmM were $5^{\prime}$ TGCTGACCTTGTCTCTGGCC3'/5'TAGTCTGGGTGGGGAATGCA3', 5'CA TTCCAGGCCATTTATGGC3'/5'GGCAAGCTTGCTTTTTGATCC $3^{\prime}$ and $5^{\prime}$ ATGGA GGTCTGCTGGTCC3'/5'TCCAGGAGCTGTAGGGGG3', respectively.

Flow cytometry. Cell populations were analyzed for cell surface marker expression, pentamer staining and/or intracellular expression of gzmA and gzmB by FACS as described. ${ }^{37}$ In all cases, cells were incubated with anti $(\alpha)$-FcR-antibody (clone 2.4G2) prior to the first staining step. Detection of LCMV-immune CTL was conducted by staining MACS-selected $\mathrm{CD} 8^{+} \mathrm{T}$ cells with gp33-labeled pentamers (H-2Db pent-PE). Rabbit immune serum (IS) specific for mouse gzmA $\left(\alpha\right.$-mgzmA) has been described. ${ }^{37}$ Rabbit $\alpha$-mouse gzmB ( $\alpha$-mgzmB) IS was generated by repeated immunization of rabbits with pro-rec.gzmB as described for $\alpha$-mgzmA IS.

Lamp-1 mobilization assay. Lamp-1 (CD107a) expression in the cell membrane of ex vivo CTLs was analyzed as described. ${ }^{38}$ Briefly, MEF cells were pretreated with the LCMV-immunodominant peptide gp33 for $2 \mathrm{~h}$ prior to incubation with ex vivo-derived LCMV-immune CTL at 2:1 E:T cell ratio (triplicates, $4 \times 10^{4}$ targets and $8 \times 10^{4} \mathrm{CTLS} /$ well). Subsequently, the mixed cell population was treated with $1 \mu \mathrm{l}$ (final dilution 1:200) of $\alpha$-Lamp-1-FITC antibody (BD Pharmingen) in the presence of monensin for $6 \mathrm{~h}$ at $37^{\circ} \mathrm{C}, 7 \% \mathrm{CO}_{2}$. Non-attached cells were collected, labeled with $\alpha$-CD8-APC antibody and analyzed by FACS.

Analysis of pro-apoptotic processes. Cell death induced by ex vivo CTL in MEF cells was analyzed as described. ${ }^{6}$ MEF cells were pretreated with the LCMV-immunodominant peptide gp33 for $2 \mathrm{~h}$ prior to incubation with ex vivo-derived LCMV-immune CTL at 10:1 E:T cell ratio for $4 \mathrm{~h}$ at $37^{\circ} \mathrm{C}, 7 \% \mathrm{CO}_{2}{ }^{6}$ Subsequently, different apoptotic parameters were tested in the target population (CD8 ${ }^{-}$) by FACS with a FACScan (BD Pharmingen) and CellQuest ${ }^{\circledR}$ software as follows:

Cell membrane and mitochondrial membrane perturbations: PS exposure and $\mathrm{PI}$ uptake were analyzed by FACS as described ${ }^{6}$ using the annexin-V-FITC kit from $\mathrm{BD}$ Pharmingen. The mitochondrial membrane potential was measured with the fluorescent probe 3,3'-dihexyloxacarbocyanine iodide $\left(\mathrm{DiOC}_{6}(3)\right.$; Molecular Probes) and ROS generation with 2-hydroxyethidium (2-HE; Molecular Probes) as described. ${ }^{6}$

Caspase-3 activation: Caspase-3 activation was analyzed by FACS as described. ${ }^{6}$ Cells were fixed with $4 \%$ paraformaldehyde (PFA) and incubated with an FITC-labeled monoclonal antibody against the active form of caspase-3 (clone C92605; BD Pharmingen) diluted in $0.1 \%$ saponin in PBS. After two washes with $0.1 \%$ saponin in PBS, cells were resuspended in $1 \%$ PFA/PBS and analyzed by FACS.

Cytochrome $c$ release: Cytochrome $c$ release was quantified by FACS analysis as recently described. ${ }^{39}$ Briefly, MEFs $\left(1 \times 10^{6}\right)$ were mildly permeabilized with $25 \mu \mathrm{g} / \mathrm{ml}$ digitonin plus $100 \mathrm{mM} \mathrm{KCl}$ on ice for $5 \mathrm{~min}$. This leads to loss of cytosolic cytochrome c. Cells were washed once with cold PBS, fixed in 4\% PFA, permeabilized with $0.05 \%$ saponin and $3 \%$ BSA and then incubated with the $\alpha$ cytochrome $c \mathrm{mAb} 6 \mathrm{H} 2 . \mathrm{B} 4$ (BD Pharmingen) or with mouse IgG isotype as control (Jackson) followed by $\alpha$-mouse-Alexa 544 secondary antibody (Invitrogen). Cells were resuspended in $100 \mu \mathrm{l}$ PFA in PBS and analyzed by FACS.

Conformational change of Bax and Bak. Bak and Bax conformational change was analyzed in target cell populations as described. ${ }^{40}$ Briefly, MEFs were fixed in $4 \%$ PFA, permeabilized with $0.1 \%$ saponin in PBS $/ 5 \%$ FCS and incubated with polyclonal rabbit $\alpha$-Bak (NT; Upstate Biotechnology), polyclonal rabbit $\alpha$-Bax (NT; Upstate Biotechnology) or purified rabbit lgG (control). After washing $(2 \times)$ with $0.1 \%$ saponin in PBS, cells were incubated with $\alpha$-rabbit-Alexa 488 antibody (Invitrogen) in $0.1 \%$ saponin/PBS/5\% FCS, washed twice in $0.1 \%$ saponin/PBS, resuspended in $1 \%$ PFA/PBS and analyzed by FACS.

Analysis of Bid cleavage. MEF.wt cells were pretreated with the LCMVimmunodominant peptide gp33 for $2 \mathrm{~h}$ prior to incubation with ex vivo-derived LCMV-immune CTL at 10:1 $\mathrm{E}: \mathrm{T}$ cell ratio for $1 \mathrm{~h}$ at $37^{\circ} \mathrm{C}, 7 \% \mathrm{CO}_{2} .{ }^{6}$ Subsequently, cells were washed twice in cold PBS and lysed in a buffer containing $1 \%$ Triton $\mathrm{X}-100$, a protease inhibitor cocktail (Roche) and $100 \mu \mathrm{M} \mathrm{DCl}$ at $4^{\circ} \mathrm{C}$ during $1 \mathrm{~h}$. Soluble protein fractions were recovered by centrifugation, separated by SDS polyacrylamide gel electrophoresis (15\%) and transferred onto PVDF membranes. Bid was analyzed by using a rat anti-Bid Ab (a generous gift of David Huang) that recognizes both the non-cleaved $(22 \mathrm{kDa})$ and the truncated protein (tBid; $16 \mathrm{kDa})$.

Survival assays. Survival of target cells following their incubation with CTL was analyzed as described. ${ }^{27}$ This assay gives similar results to clonogenic survival assay in agar plates (AM, unpublished observations). Accordingly, MEF cells were pretreated with the LCMV-immunodominant peptide gp33 $(2 \mathrm{~h})$ prior to incubation with ex vivo-derived LCMV-immune CTLs at 10:1 E:T cell ratio for $4 \mathrm{~h}$. Wells were washed once with MEM plus 2\% FCS to remove non-adherent cells and attached cells were resuspended in MEM plus $2 \% \mathrm{FCS}$. No viable cells were detected among the non-adherent cells. Target cell survival was quantified by ${ }^{3} \mathrm{H}$-thymidine incorporation during the next $24 \mathrm{~h}$ as described. ${ }^{27}$

Acknowledgements. We thank Anton Grubisic, Aynur Ekiciler and Thomas Stehle for expert technical assistance. We also thank Pierre Golstein for his critical comments and stimulating discussions, Lars Joeckel for reading the manuscript and David Huang for the antiBid antibody. JP was supported by the Alexander von Humboldt foundation and by a research contract from Aragon I + D. 
1. Russell JH, Ley TJ. Lymphocyte-mediated cytotoxicity. Annu Rev Immunol 2002; 20 323-370.

2. Simon MM, Kramer MD. Granzyme A. Methods Enzymol 1994; 244: 68-79.

3. Tschopp J. Granzyme B. Methods Enzymol 1994; 244: 80-87.

4. Lieberman J. The ABCs of granule-mediated cytotoxicity: new weapons in the arsenal. Nat Rev Immunol 2003; 3: 361-370.

5. Trapani JA, Sutton VR. Granzyme B: pro-apoptotic, antiviral and antitumor functions. Curr Opin Immunol 2003; 15: 533-543.

6. Pardo J, Bosque A, Brehm R, Wallich R, Naval J, Mullbacher A et al. Apoptotic pathways are selectively activated by granzyme $A$ and/or granzyme $B$ in CTL-mediated target cell lysis. J Cell Biol 2004; 167: 457-468.

7. Thomas DA, Scorrano L, Putcha GV, Korsmeyer SJ, Ley TJ. Granzyme B can cause mitochondrial depolarization and cell death in the absence of BID, BAX, and BAK. Proc Natl Acad Sci USA 2001; 98: 14985-14990.

8. Bleackley RC. A molecular view of cytotoxic T lymphocyte induced killing. Biochem Cell Biol 2005; 83: 747-751.

9. Ricci JE, Munoz-Pinedo C, Fitzgerald P, Bailly-Maitre B, Perkins GA, Yadava N et al. Disruption of mitochondrial function during apoptosis is mediated by caspase cleavage of the p75 subunit of complex I of the electron transport chain. Cell 2004; 117 773-786.

10. Simizu S, Takada M, Umezawa K, Imoto M. Requirement of caspase-3(-like) protease mediated hydrogen peroxide production for apoptosis induced by various anticancer drugs. J Biol Chem 1998; 273: 26900-26907.

11. Savill J, Fadok V. Corpse clearance defines the meaning of cell death. Nature 2000; 407 784-788

12. Waterhouse NJ, Sedelies KA, Sutton VR, Pinkoski MJ, Thia KY, Johnstone R et al. Functional dissociation of DeltaPsim and cytochrome $c$ release defines the contribution of mitochondria upstream of caspase activation during granzyme B-induced apoptosis. Cell Death Differ 2006; 13: 607-618.

13. Adrain C, Murphy BM, Martin SJ. Molecular ordering of the caspase activation cascade initiated by the cytotoxic T lymphocyte/natural killer (CTL/NK) protease granzyme B. J Biol Chem 2005; 280: 4663-4673.

14. Adrain C, Duriez PJ, Brumatti G, Delivani P, Martin SJ. The cytotoxic lymphocyte protease, granzyme $B$, targets the cytoskeleton and perturbs microtubule polymerization dynamics. J Biol Chem 2006; 281: 8118-8125.

15. Goping IS, Sawchuk T, Underhill DA, Bleackley RC. Identification of \{alpha\}-tubulin as a granzyme B substrate during CTL-mediated apoptosis. J Cell Sci 2006; 119: 858-865.

16. Bredemeyer AJ, Townsend RR, Ley TJ. Use of protease proteomics to discover granzyme B substrates. Immunol Res 2005; 32: 143-153.

17. Waterhouse NJ, Sedelies KA, Trapani JA. Role of Bid-induced mitochondrial oute membrane permeabilization in granzyme B-induced apoptosis. Immunol Cell Biol 2006; 84 72-78.

18. Scaffidi C, Fulda S, Srinivasan A, Friesen C, Li F, Tomaselli KJ et al. Two CD95 (APO-1/ Fas) signaling pathways. EMBO J 1998; 17: 1675-1687.

19. Green DR, Kroemer G. The pathophysiology of mitochondrial cell death. Science 2004 305: 626-629.

20. Martinvalet D, Zhu P, Lieberman J. Granzyme A induces caspase-independent mitochondrial damage, a required first step for apoptosis. Immunity 2005; 22: 355-370.

21. Metkar SS, Wang B, Ebbs ML, Kim JH, Lee YJ, Raja SM et al. Granzyme B activates procaspase- 3 which signals a mitochondrial amplification loop for maximal apoptosis. J Cell Biol 2003; 160: 875-885.
22. Lakhani SA, Masud A, Kuida K, Porter Jr GA, Booth CJ, Mehal WZ et al. Caspases 3 and 7 : key mediators of mitochondrial events of apoptosis. Science 2006; 311: 847-851.

23. Waterhouse NJ, Sedelies KA, Browne KA, Wowk ME, Newbold A, Sutton VR et al. A central role for Bid in granzyme B-induced apoptosis. J Biol Chem 2005; 280: 4476-4482.

24. Casciola-Rosen L, Garcia-Calvo M, Bull HG, Becker JW, Hines T, Thornberry NA et al. Mouse and human granzyme $B$ have distinct tetrapeptide specificities and abilities to recruit the bid pathway. J Biol Chem 2007; 282: 4545-4552.

25. Cullen SP, Adrain C, Luthi AU, Duriez PJ, Martin SJ. Human and murine granzyme B exhibit divergent substrate preferences. J Cell Biol 2007; 176: 435-444.

26. Kaiserman D, Bird CH, Sun J, Matthews A, Ung K, Whisstock JC et al. The major human and mouse granzymes are structurally and functionally divergent. J Cell Biol 2006; 175: $619-630$

27. Pardo J, Balkow S, Anel A, Simon MM. Granzymes are essential for natural killer cellmediated and perf-facilitated tumor control. Eur J Immunol 2002; 32: 2881-2887.

28. Revell PA, Grossman WJ, Thomas DA, Cao X, Behl R, Ratner JA et al. Granzyme B and the downstream granzymes $\mathrm{C}$ and/or $\mathrm{F}$ are important for cytotoxic lymphocyte functions. $\mathrm{J}$ Immunol 2005; 174: 2124-2131.

29. Waterhouse NJ, Sutton VR, Sedelies KA, Ciccone A, Jenkins M, Turner SJ et al. Cytotoxic $T$ lymphocyte-induced killing in the absence of granzymes $A$ and $B$ is unique and distinct from both apoptosis and perforin-dependent lysis. J Cell Biol 2006; 173: 133-144.

30. Kothakota S, Azuma T, Reinhard C, Klippel A, Tang J, Chu K et al. Caspase-3-generated fragment of gelsolin: effector of morphological change in apoptosis. Science 1997; 278: 294-298.

31. Slee EA, Adrain C, Martin SJ. Executioner caspase-3, -6, and -7 perform distinct, nonredundant roles during the demolition phase of apoptosis. J Biol Chem 2001; 276 : 7320-7326.

32. Sebbagh M, Hamelin J, Bertoglio J, Solary E, Breard J. Direct cleavage of ROCK II by granzyme $B$ induces target cell membrane blebbing in a caspase-independent manner. $J$ Exp Med 2005; 201: 465-471

33. Han J, Goldstein LA, Gastman BR, Rabinovitz A, Rabinowich H. Disruption of Mcl-1.Bim complex in granzyme B-mediated mitochondrial apoptosis. J Biol Chem 2005; 280: 16383-16392.

34. MacDonald G, Shi L, Vande Velde C, Lieberman J, Greenberg AH. Mitochondriadependent and -independent regulation of granzyme B-induced apoptosis. $J$ Exp Med 1999; 189: 131-144.

35. Sutton VR, Davis JE, Cancilla M, Johnstone RW, Ruefli AA, Sedelies K et al. Initiation of apoptosis by granzyme $B$ requires direct cleavage of bid, but not direct granzyme B-mediated caspase activation. J Exp Med 2000; 192: 1403-1414.

36. Müllbacher A, Waring P, Hia RT, Tran T, Chin S, Stehle T et al. Granzymes are the essential downstream effector molecules for the control of primary virus infections by cytolytic leukocytes. Proc Natl Acad Sci USA 1999; 96: 13950-13955.

37. Martin P. Wallich R, Pardo J, Mullbacher A, Munder M, Modolell M et al. Quiescent and activated mouse granulocytes do not express granzyme $A$ and $B$ or perforin: similarities or differences with human polymorphonuclear leukocytes? Blood 2005; 106: 2871-2878.

38. Rubio V, Stuge TB, Singh N, Betts MR, Weber JS, Roederer M et al. Ex vivo identification, isolation and analysis of tumor-cytolytic T cells. Nat Med 2003; 9: 1377-1382.

39. Waterhouse NJ, Trapani JA. A new quantitative assay for cytochrome $c$ release in apoptotic cells. Cell Death Differ 2003; 10: 853-855.

40. Pardo J, Urban C, Galvez EM, Ekert PG, Muller U, Kwon-Chung J et al. The mitochondrial protein Bak is pivotal for gliotoxin-induced apoptosis and a critical host factor of Aspergillus fumigatus virulence in mice. J Cell Biol 2006; 174: 509-519.

\section{Supplementary Information accompanies the paper on Cell Death and Differentiation website (http://www.nature.com/cdd)}

